\title{
CONJUGATE HEAT AND MASS TRANSFER ON FLUCTUATING MIXED CONVECTION FLOW ALONG A VERTICAL WEDGE WITH THERMAL RADIATION
}

\author{
M.N. FIROZA, N.C. ROY and Md. A. HOSSAIN* \\ Department of Mathematics, University of Dhaka \\ Dhaka 1000, BANGLADESH \\ E-mail: anwar.cfd@gmail.com
}

\begin{abstract}
We study the boundary layer characteristics of heat and mass transfer flow past a vertical wedge in the presence of thermal radiation. The surface temperature and the species concentration are assumed to be oscillating in the magnitude but not in the direction of oncoming flow velocity. The governing equations have been solved by two distinct methods, namely, the straightforward finite difference method for the entire frequency range, and the series solution for the low frequency range and the asymptotic series expansion method for the high frequency range. Numerical solutions have been presented in terms of the amplitudes and phase angles of the skin friction, the rate of heat transfer and the mass transfer with the variations of Richardson's number, the Prandtl number, the conduction-radiation parameter, the surface temperature parameter and the Schmidt number. Furthermore, the effects of these parameters are examined in terms of the transient skin friction, heat transfer and mass transfer.
\end{abstract}

Key words: mixed convection, thermal radiation, vertical wedge.

\section{Introduction}

The combined effect of both free and forced convection is known as mixed convection and has been the focus of research because of its various applications in thermal engineering and science, such as, compact heat exchangers, boilers, atmospheric boundary layer flows, solar energy systems and cooling of electronic devices. The effect of mixed convection must be considered in a mathematical model when the forced and the free convection effects are of comparable magnitude in a system. In other words, mixed convection takes place when the influence of buoyancy forces on a forced flow or vice versa is significant. There are numerous studies on mixed convection boundary layer flow subject to different circumstances such as vertical/inclined/horizontal plate or wedge surfaces in a fluid.

Due to the occurrence in many interesting and important fluid-mechanical problems, oscillating flow and heat transfer under the influence of free-stream oscillation have been the important topic of research, for example, the accelerating and decelerating phases of missile flight, the intermittent flow in an engine during unstable combustion, heat transfer process frequently experienced in liquid rocket and turbo-jet engines and thermal failure of the resonance tube heating in which the effect of heat generation appears to be significant. Lighthill [1] first initiated the investigation of how a boundary layer responds to fluctuations of the external velocity about a steady mean.

Sing et al. [2] studied the unsteady boundary layer characteristics of the mixed convection flow over a vertical wedge with constant suction or injection. Yih [3] and Watanabe [4] focused on the forced convection boundary-layer flow over a wedge taking into account the effect of uniform suction and blowing. Kafoussias and Nanousis [5] numerically investigated the behavior of a laminar boundary layer flow over a wedge with suction/injection. Devi and Kandasamy [6] presented the influences of thermal stratification as

\footnotetext{
* To whom correspondence should be addressed
} 
well as mass transfer over a porous wedge. Gersten [7] theoretically investigated the time-mean heat transfer in a wedge-type flow with small amplitude oscillation and found that that the time-mean heat-transfer rate is smaller than that without oscillation. Kumari and Gorla [8] carried out a boundary layer analysis considering the combined convection along a vertical non-isothermal wedge situated in a fluid-saturated porous medium. Hossain et al. [9] studied the effect of forced flow of an incompressible fluid past a horizontal wedge subject to heat flux boundary condition. Kumari et al. [10] examined the magnetohydrodynamic mixed convection flow over a vertical porous wedge. On the contrary, Kandasamy et al. [11] investigated the effects of variable viscosity and thermophoresis of an electrically conducting fluid for mixed convective heat and mass transfer past a porous wedge in the presence of chemical reaction.

It is worthwhile to note that thermal radiation could be a controlling factor in many technological problems, for example, liquid metal fluids, cooling of nuclear reactors, power generation systems and high temperature plasmas. Uddin et al. [12] investigated the effects of thermal radiation and heat generation/absorption on MHD heat transfer flow of a micropolar fluid past a wedge. Yih [13] studied the influence of radiation on a mixed convection flow about an isothermal wedge kept in a saturated porous medium. Al-Odat et al. [14] examined the mixed convection flow from an isothermal wedge in the presence of thermal radiation. Chamkha et al. [15] numerically observed the hydromagnetic forced convective boundary-layer flow considering the effects of heat-generation or absorption and thermal radiation over a non-isothermal wedge. Elbashabeshy and Dimian [16] studied the effect of radiation on the behavior of the flow and heat transfer over a wedge.

Now there are many transport processes which frequently occur both in nature and in industries with the combined heat and mass transfer. As a result, heat and mass transfer problems in the presence of thermal radiation have attracted attention due to many practical applications such as drying, distribution of temperature and moisture over agricultural fields and groves of fruit trees, damage of crops due to freezing, evaporation at the surface of water body, energy transfer in a wet cooling tower and flow in a desert cooler. However previous studies have simplified the radiation term by assuming changes in the Prandtl number. In this study, we investigate the unsteady laminar boundary layer mixed convective heat and mass transfer of a viscous incompressible fluid past a vertical wedge in the presence of thermal radiation. The nonlinear effect of temperature on radiation might be captured from this analysis. The influences of Richardson's number, Ri, which is introduced to measure the effect of mixed convection, the Prandtl number, Pr, the conductionradiation parameter, $R_{d}$, the surface temperature parameter $\Delta$ and the Schmidt number, $S c$ on the amplitudes and phase angles of skin-friction, heat transfer and mass transfer have been presented. In addition, the variations of the transient skin-friction, heat transfer and mass transfer are observed for different values of $\operatorname{Pr}$ and $R_{d}$.

\section{Mathematical formulation}

A two-dimensional, unsteady, laminar mixed convection boundary layer flow of a viscous incompressible fluid past a wedge taking into account thermal radiation is considered. The physical configuration and coordinate system of the problem is shown in Fig.1. The surface temperature of the wedge is assumed to be oscillating with small amplitude about a constant mean temperature.

Under the usual Boussinesq approximation, the conservation equations for the unsteady, laminar, two dimensional boundary layer flow problem can be written as

$$
\begin{aligned}
& \frac{\partial u}{\partial x}+\frac{\partial v}{\partial y}=0 \\
& \frac{\partial u}{\partial t}+u \frac{\partial u}{\partial x}+v \frac{\partial u}{\partial y}=\frac{\partial U}{\partial t}+U \frac{\partial U}{\partial x}+v \frac{\partial^{2} u}{\partial y^{2}}+g\left\{\beta_{T}\left(T-T_{\infty}\right)+\beta_{C}\left(C-C_{\infty}\right)\right\} \cos (\pi / 4),
\end{aligned}
$$




$$
\begin{aligned}
& \frac{\partial T}{\partial t}+u \frac{\partial T}{\partial x}+v \frac{\partial T}{\partial y}=\alpha\left(\frac{\partial^{2} T}{\partial y^{2}}-\frac{1}{\kappa} \frac{\partial q_{r}}{\partial y}\right), \\
& \frac{\partial C}{\partial t}+u \frac{\partial C}{\partial x}+v \frac{\partial C}{\partial y}=D \frac{\partial^{2} C}{\partial y^{2}} .
\end{aligned}
$$

In the above equations, $x$ and $y$ are the coordinates parallel with and perpendicular to the wedge surface, $u$ and $v$ the velocity components in the $x$ - and $y$-directions, respectively, $v$ is the coefficient of viscosity, $g$ is the acceleration due to gravity, $\beta_{T}$ and $\beta_{C}$ are the coefficient of volumetric expansion for temperature and concentration, respectively, $\alpha$ is the thermal diffusivity, $\alpha$ is the thermal diffusivity and $D$ is the molecular diffusivity of the species. Furthermore, $T$ and $T_{\infty}$ are the temperature of the fluid in the boundary layer and the ambient fluid, respectively, while $C$ and $C_{\infty}$ are the species concentration and the ambient concentration.

The boundary conditions are

$$
\begin{aligned}
& u=v=0, T=T_{\infty}+\left(T_{w}-T_{\infty}\right)\left(1+\varepsilon e^{i \omega t}\right), C=C_{\infty}+\left(C_{w}-C_{\infty}\right)\left(1+\varepsilon e^{i \omega t}\right) \quad \text { at } y=0 \\
& u=U(x, t), \quad T=T_{\infty}, \quad C=C_{\infty} \quad \text { as } \quad y \rightarrow \infty .
\end{aligned}
$$

Here $T_{w}$ is the mean surface temperature with $T_{w}>T_{\infty}$ and $C_{w}$ is the mean surface concentration with $C_{w}>C_{\infty}$. The free-stream velocity $U(x, t)$ is taken as

$$
U(x, t)=U_{0} x^{1 / 2}\left(1+\varepsilon e^{i \omega t}\right)
$$

where $U_{0}, \varepsilon$ are constant and $\omega$ is the frequency.

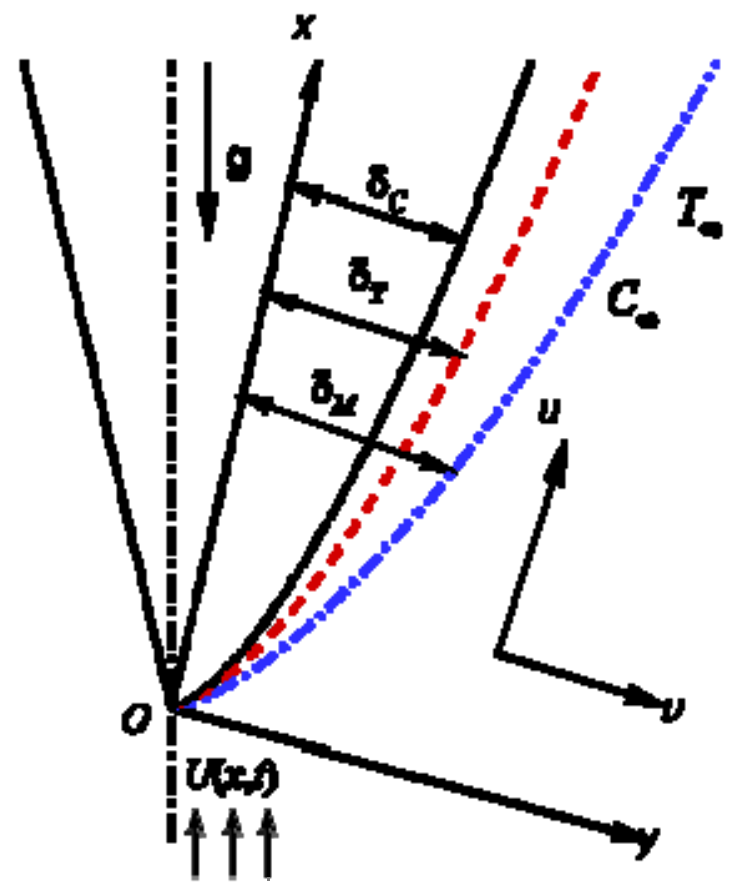

Fig.1. Flow configuration and coordinate system. 
In Eq.(2.3), the quantity $q_{r}$ is the radiative heat-flux in the $y$-direction. We assume that the fluid is gray and optically thick as if it could only absorb and emit radiation. Under consideration of an optically thick boundary layer, the radiative heat-flux term may be simplified using the Rosseland diffusion approximation and is defined as

$$
q_{r}=-\frac{4 \sigma}{3 \kappa^{*}} \frac{\partial T^{4}}{\partial y}
$$

where $k^{*}$ is the mean absorption coefficient and $\sigma$ is the Stefan-Boltzman constant.

Now we define the stream function, $\psi$, as

$$
u=\frac{\partial \psi}{\partial y}, \quad v=-\frac{\partial \psi}{\partial x}
$$

which satisfies the continuity Eq.(2.1). forms

From the boundary conditions (2.5), the functions $\psi, T$ and $C$ may be considered in the following

$$
\begin{aligned}
& \psi(x, y, t)=\psi_{s}(x, y)+\varepsilon \psi_{l}(x, y) e^{i \omega t} \\
& T(x, y, t)=T_{s}(x, y)+\varepsilon T_{1}(x, y) e^{i \omega t} \\
& C(x, y, t)=C_{S}(x, y)+\varepsilon C_{1}(x, y) e^{i \omega t}
\end{aligned}
$$

where $\varepsilon<<1$ is a constant, $\psi_{s}, T_{s}$ and $C_{s}$ are steady components and $\psi_{1}, T_{1}$ and $C_{1}$ are time-dependent components.

Substituting (2.7)-(2.10) into (2.2)-(2.4) and equating the coefficients of $\varepsilon^{0}$, one obtains

$$
\begin{aligned}
& \frac{\partial \psi_{s}}{\partial y} \frac{\partial^{2} \psi_{s}}{\partial x \partial y}-\frac{\partial \psi_{s}}{\partial x} \frac{\partial^{2} \psi_{s}}{\partial y^{2}}=\frac{1}{2} U_{0}^{2}+v \frac{\partial^{3} \psi_{s}}{\partial y^{3}}+g\left\{\beta_{T}\left(T_{s}-T_{\infty}\right)+\beta_{C}\left(C_{s}-C_{\infty}\right)\right\} \cos (\pi / 4) \\
& \frac{\partial \psi_{s}}{\partial y} \frac{\partial T_{s}}{\partial x}-\frac{\partial \psi_{s}}{\partial x} \frac{\partial T_{s}}{\partial y}=\alpha \frac{\partial^{2} T_{s}}{\partial y^{2}}+\frac{16 \alpha \sigma}{3 \kappa\left(a+\sigma_{s}\right)} \frac{\partial}{\partial y}\left(T_{s}^{3} \frac{\partial T_{s}}{\partial y}\right) \\
& \frac{\partial \psi_{s}}{\partial y} \frac{\partial C_{s}}{\partial x}-\frac{\partial \psi_{s}}{\partial x} \frac{\partial C_{s}}{\partial y}=D \frac{\partial^{2} C_{s}}{\partial y^{2}}
\end{aligned}
$$

To render the above equations in an dimensionless form, the following functions and variables are introduced 


$$
\begin{aligned}
& \psi_{s}=\left(v U_{0}\right)^{1 / 2}(1+\operatorname{Pr})^{-3 / 4} x^{3 / 4} f(\eta), \quad T_{s}=T_{\infty}+\left(T_{w}-T_{\infty}\right) \theta(\eta), \\
& C_{s}=C_{\infty}+\left(C_{w}-C_{\infty}\right) \varphi(\eta), \quad \eta=\left(\frac{U_{0}}{v}\right)^{1 / 2}(1+\operatorname{Pr})^{1 / 4} x^{-1 / 4} y .
\end{aligned}
$$

Using Eqs (2.14) in Eqs (2.11)-(2.13), we obtain

$$
\begin{aligned}
& (1+\operatorname{Pr}) f^{\prime \prime \prime}+\frac{3}{4} f f^{\prime \prime}-\frac{1}{2}\left\{f^{\prime 2}-(1+\operatorname{Pr})\right\}+(1+\operatorname{Pr})(1-w) \operatorname{Ri} \theta+(1+\operatorname{Pr}) w \operatorname{Ri} \varphi=0, \\
& \frac{(1+\operatorname{Pr})}{\operatorname{Pr}}\left\{1+\frac{4}{3} R_{d}(1+\Delta \theta)^{3}\right\} \theta^{\prime \prime}+\frac{4(1+\operatorname{Pr}) \Delta}{\operatorname{Pr}} R_{d}(1+\Delta \theta)^{2} \theta^{\prime 2}+\frac{3}{4} f \theta^{\prime}=0, \\
& \frac{(1+\operatorname{Pr})}{\operatorname{Sc}} \varphi^{\prime \prime}+\frac{3}{4} f \varphi^{\prime}=0,
\end{aligned}
$$

subject to the boundary conditions

$$
\begin{aligned}
& f=f^{\prime}=0, \theta=1, \varphi=1 \quad \text { at } \quad \eta=0, \\
& f^{\prime}=(1+\operatorname{Pr})^{1 / 2}, \theta=0, \varphi=0 \quad \text { as } \quad \eta \rightarrow \infty .
\end{aligned}
$$

Again substituting the expressions (2.7)-(2.10) into Eqs (2.2)-(2.4) and equating the coefficients of $\varepsilon$, one can find

$$
\begin{aligned}
& i \omega \frac{\partial \psi_{1}}{\partial y}+\frac{\partial \psi_{0}}{\partial y} \frac{\partial^{2} \psi_{1}}{\partial x \partial y}+\frac{\partial \psi_{1}}{\partial y} \frac{\partial^{2} \psi_{s}}{\partial x \partial y}-\frac{\partial \psi_{s}}{\partial x} \frac{\partial^{2} \psi_{1}}{\partial y^{2}}-\frac{\partial \psi_{1}}{\partial x} \frac{\partial^{2} \psi_{s}}{\partial y^{2}}= \\
& =i \omega U_{0} x^{1 / 2}+U_{0}^{2}+v \frac{\partial^{3} \psi_{1}}{\partial y^{3}}+g\left(\beta_{T} T_{1}+\beta_{C} C_{1}\right) \cos (\pi / 4), \\
& i \omega T_{1}+\frac{\partial \psi_{s}}{\partial y} \frac{\partial T_{1}}{\partial x}+\frac{\partial \psi_{1}}{\partial y} \frac{\partial T_{s}}{\partial x}-\frac{\partial \psi_{s}}{\partial x} \frac{\partial T_{1}}{\partial y}-\frac{\partial \psi_{1}}{\partial x} \frac{\partial T_{s}}{\partial y}= \\
& =\alpha \frac{\partial^{2} T_{1}}{\partial y^{2}}+\frac{16 \alpha \sigma}{3 \kappa\left(a+\sigma_{s}\right)} \frac{\partial}{\partial y}\left(T_{s}^{3} \frac{\partial T_{1}}{\partial y}+3 T_{s}^{2} T_{1} \frac{\partial T_{s}}{\partial y}\right), \\
& i \omega C_{1}+\frac{\partial \psi_{s}}{\partial y} \frac{\partial C_{1}}{\partial x}+\frac{\partial \psi_{1}}{\partial y} \frac{\partial C_{s}}{\partial x}-\frac{\partial \psi_{s}}{\partial x} \frac{\partial C_{1}}{\partial y}-\frac{\partial \psi_{1}}{\partial x} \frac{\partial C_{s}}{\partial y}=D \frac{\partial^{2} C_{1}}{\partial y^{2}} .
\end{aligned}
$$

The associated boundary conditions are

$$
\psi_{1}=\frac{\partial \psi_{1}}{\partial y}=0, \quad T_{1}=T_{w}-T_{\infty}, \quad C_{1}=C_{w}-C_{\infty} \quad \text { at } \quad y=0
$$




$$
\frac{\partial \psi_{1}}{\partial y}=U_{0} x^{1 / 2}, \quad T_{1}=0, \quad C_{1}=0 \quad \text { as } \quad y \rightarrow \infty .
$$

To non-dimensionalize Eqs (2.20)-(2.22), we define the following functions

$$
\begin{aligned}
& \psi_{1}=\left(v U_{0}\right)^{1 / 2}(1+\operatorname{Pr})^{-3 / 4} x^{3 / 4} F(\xi, \eta), \quad T_{1}=T_{\infty}+\left(T_{w}-T_{\infty}\right) \Theta(\xi, \eta), \\
& C_{1}=C_{\infty}+\left(C_{w}-C_{\infty}\right) \Phi(\xi, \eta) .
\end{aligned}
$$

Using Eqs (2.14) and (2.25) in Eqs (2.20)-(2.22), we obtain

$$
\begin{aligned}
& (1+\operatorname{Pr}) F^{\prime \prime \prime}+\frac{3}{4}\left(f F^{\prime \prime}+f^{\prime \prime} F\right)-f^{\prime} F^{\prime}+i \xi\left\{(1+\operatorname{Pr})-\sqrt{1+\operatorname{Pr}} F^{\prime}\right\}+(1+\operatorname{Pr})(1-w) \operatorname{Ri} \Theta+ \\
& +(1+\operatorname{Pr}) w \operatorname{Ri} \Phi+(1+\operatorname{Pr})=\frac{1}{2} \xi\left(f^{\prime} \frac{\partial F^{\prime}}{\partial \xi}-f^{\prime \prime} \frac{\partial F}{\partial \xi}\right), \\
& \frac{(1+\operatorname{Pr})}{\operatorname{Pr}}\left\{1+\frac{4}{3} R_{d}(1+\Delta \theta)^{3}\right\} \Theta^{\prime \prime}+\frac{8(1+\operatorname{Pr}) \Delta}{\operatorname{Pr}} R_{d}(1+\Delta \theta)^{2} \theta^{\prime} \Theta^{\prime}+ \\
& +\frac{8(1+\operatorname{Pr}) \Delta^{2}}{\operatorname{Pr}} R_{d}(1+\Delta \theta) \theta^{\prime 2} \Theta+\frac{4(1+\operatorname{Pr}) \Delta}{\operatorname{Pr}} R_{d}(1+\Delta \theta)^{2} \theta^{\prime \prime} \Theta+ \\
& +\frac{3}{4}\left(F \theta^{\prime}+f \Theta^{\prime}\right)-i \xi \sqrt{1+\operatorname{Pr} \Theta}=\frac{1}{2} \xi\left(f^{\prime} \frac{\partial \Theta}{\partial \xi}-\theta^{\prime} \frac{\partial F}{\partial \xi}\right), \\
& \frac{(1+\operatorname{Pr})}{\operatorname{Sc}} \Phi^{\prime \prime}+\frac{3}{4}\left(f \Phi^{\prime}+\theta^{\prime} F\right)-i \xi \sqrt{1+\operatorname{Pr}} \Phi=\frac{1}{2} \xi\left(f^{\prime} \frac{\partial \Phi}{\partial \xi}-\varphi^{\prime} \frac{\partial F}{\partial \xi}\right) .
\end{aligned}
$$

Here $\xi=\omega x^{1 / 2} / U_{0}, \mathrm{Gr}_{x}=\mathrm{Gr}_{T}+\mathrm{Gr}_{C}=g\left\{\beta_{T}\left(T_{w}-T_{\infty}\right)+\beta_{C}\left(C_{w}-C_{\infty}\right)\right\} x^{3} \cos (\pi / 4) / v^{2}$ is the global Grashof number where $\mathrm{Gr}_{T}$ is the Grashof number for thermal diffusion and $\mathrm{Gr}_{C}$ is the Grashof number for mass diffusion, $\mathrm{Re}_{x}=U_{0} x^{3 / 2} / v$ is the Reynolds number, $\mathrm{Ri}=\mathrm{Gr}_{x} / \mathrm{Re}_{x}{ }^{2}$ is Richardson's number, $\mathrm{Pr}=v / \alpha$ is the Prandtl number, $\mathrm{Sc}=v / D$ is the Schmidt number. Moreover, $R_{d}=4 \sigma T_{\infty}^{3} / \kappa\left(a+\sigma_{s}\right)$ is the Planck constant or the conduction radiation parameter and $\Delta=T_{w} / T_{\infty}-1$ is the surface temperature parameter.

The corresponding boundary conditions become

$$
\begin{aligned}
& F=F^{\prime}=0, \quad \Theta=1, \quad \Phi=1 \quad \text { at } \quad \eta=0, \\
& F^{\prime}=(1+\operatorname{Pr})^{1 / 2}, \quad \Theta=0, \quad \Phi=0 \quad \text { as } \quad \eta \rightarrow \infty .
\end{aligned}
$$

Once the solutions of Eqs (2.15)-(2.19) and (2.26)-(2.30) are known, we can easily determine the values of the physical quantities, namely, the skin friction, the rate of heat transfer and the mass transfer at the surface of the wedge. These are important not only from a physical point of view but also experimental point of view. In this study, the results are discussed in terms of the amplitudes of the skin friction, the heat transfer rate and the mass transfer rate using the relations 


$$
\begin{aligned}
& A_{u}=\left|F^{\prime \prime}(\xi, 0)\right|=\sqrt{\left\{F_{r}^{\prime \prime}(\xi, 0)\right\}^{2}+\left\{F_{i}^{\prime \prime}(\xi, 0)\right\}^{2}}, \\
& A_{q}=\left|\Theta^{\prime}(\xi, 0)\right|=\sqrt{\left\{\Theta_{r}^{\prime}(\xi, 0)\right\}^{2}+\left\{\Theta_{i}^{\prime}(\xi, 0)\right\}^{2}}, \\
& A_{m}=\left|\Phi^{\prime}(\xi, 0)\right|=\sqrt{\left\{\Phi_{r}^{\prime}(\xi, 0)\right\}^{2}+\left\{\Phi_{i}^{\prime}(\xi, 0)\right\}^{2}}
\end{aligned}
$$

where $\left(F_{r}^{\prime \prime}, F_{i}^{\prime \prime}\right),\left(\Theta_{r}^{\prime}, \Theta_{i}^{\prime}\right)$ and $\left(\Phi_{r}^{\prime}, \Phi_{i}^{\prime}\right)$ are the corresponding real and imaginary parts of the gradients of the velocity, concentration and temperature at the surface. Also, the phase angles of the skin friction, the heat transfer rate and the mass transfer rate are expressed by

$$
\phi_{u}=\tan ^{-1}\left(\frac{F_{i}^{\prime \prime}}{F_{r}^{\prime \prime}}\right), \quad \phi_{q}=\tan ^{-1}\left(\frac{\Theta_{i}^{\prime}}{\Theta_{r}^{\prime}}\right), \quad \phi_{m}=\tan ^{-1}\left(\frac{\Phi_{i}^{\prime}}{\Phi_{r}^{\prime}}\right) .
$$

\section{Methods of solution}

The set of Eqs (2.15) to (2.19) represents the steady state mean flow and temperature fields. We solve these equations by employing the straightforward finite difference method for different values of the physical parameters $\mathrm{Pr}, \mathrm{Sc}, \mathrm{Ri}, R_{d}$ and $\Delta$. The resulting solutions are then applied in finding the solutions of Eqs (2.26) to (2.30) that provide the oscillating parts of the flow and the temperature fields. With the aim of validating the numerical solutions, Eqs (2.26) to (2.30) are also solved by the extended series expansion method for small $\xi$ and the asymptotic method for large $\xi$. The solutions are discussed in the subsequent parts in details.

\subsection{Series solutions for small $\xi$}

With the purpose of obtaining the effect of mixed convection flow near the leading edge, the result based on the finite number of terms in the series is only valid in a very small range of frequencies. Thus near the leading edge, or equivalently for small $\xi$, we expand the functions $F, \Theta$ and $\Phi$ in powers of $\xi$ as given bellow

$$
\begin{aligned}
& F(\xi, \eta)=\sum_{m=0}^{\infty}(2 i \xi)^{m} F_{m}(\eta), \quad \Theta(\xi, \eta)=\sum_{m=0}^{\infty}(2 i \xi)^{m} \Theta_{m}(\eta), \\
& \Phi(\xi, \eta)=\sum_{m=0}^{\infty}(2 i \xi)^{m} \Phi_{m}(\eta) .
\end{aligned}
$$

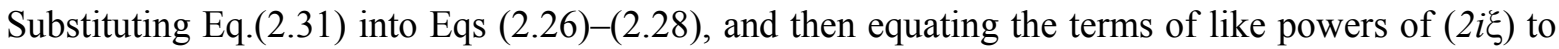
zero, the following groups of ordinary differential equations are obtained

For $m=0$

$$
\begin{aligned}
& (1+\operatorname{Pr}) F_{0}^{\prime \prime \prime}+\frac{3}{4}\left(f F_{0}^{\prime \prime}+f^{\prime \prime} F_{0}\right)-f^{\prime} F_{0}^{\prime}+ \\
& +(1-\omega)(1+\operatorname{Pr}) \operatorname{Ri}_{0}+\omega(1+\operatorname{Pr}) \operatorname{Ri} \Phi_{0}+(1+\operatorname{Pr})=0
\end{aligned}
$$




$$
\begin{aligned}
& \frac{(1+\operatorname{Pr})}{\operatorname{Pr}}\left\{1+\frac{4}{3} R_{d}(1+\Delta \theta)^{3}\right\} \Theta_{0}^{\prime \prime}+\frac{8(1+\operatorname{Pr}) \Delta}{\operatorname{Pr}} R_{d}(1+\Delta \theta)^{2} \theta^{\prime} \Theta_{0}^{\prime}+ \\
& +\frac{8(1+\operatorname{Pr}) \Delta^{2}}{\operatorname{Pr}} R_{d}(1+\Delta \theta) \theta^{\prime 2} \Theta_{0}+\frac{4(1+\operatorname{Pr}) \Delta}{\operatorname{Pr}} R_{d}(1+\Delta \theta)^{2} \theta^{\prime \prime} \Theta_{0}+\frac{3}{4}\left(F_{0} \theta^{\prime}+f \Theta_{0}^{\prime}\right)=0 \\
& \frac{1+\operatorname{Pr}}{\mathrm{Sc}} \Phi_{0}^{\prime \prime}+\frac{3}{4}\left(f \Phi_{0}^{\prime}+\varphi_{0}^{\prime} F_{0}\right)=0 .
\end{aligned}
$$

For $m=1$

$$
\begin{aligned}
& (1+\operatorname{Pr}) F_{1}^{\prime \prime \prime}+\frac{3}{4} f F_{1}^{\prime \prime}+\frac{5}{4} f^{\prime \prime} F_{1}-\frac{3}{2} f^{\prime} F_{1}^{\prime}+(1-\omega)(1+\operatorname{Pr}) \mathrm{Ri \Theta} \Theta_{1}+ \\
& +\omega(1+\operatorname{Pr}) \operatorname{Ri} \Phi_{1}+\frac{1}{2}(1+\operatorname{Pr})=\frac{1}{2} \sqrt{1+\operatorname{Pr}} F_{0}^{\prime} \\
& \frac{(1+\operatorname{Pr})}{\operatorname{Pr}}\left\{1+\frac{4}{3} R_{d}(1+\Delta \theta)^{3}\right\} \Theta_{1}^{\prime \prime}+\frac{8(1+\operatorname{Pr}) \Delta}{\operatorname{Pr}} R_{d}(1+\Delta \theta)^{2} \theta^{\prime} \Theta_{1}^{\prime}+ \\
& +\frac{8(1+\operatorname{Pr}) \Delta^{2}}{\operatorname{Pr}} R_{d}(1+\Delta \theta) \theta^{\prime 2} \Theta_{1}+\frac{4(1+\operatorname{Pr}) \Delta}{\operatorname{Pr}} R_{d}(1+\Delta \theta)^{2} \theta^{\prime \prime} \Theta_{1}+ \\
& +\frac{3}{4} f \Theta_{1}^{\prime}+\frac{5}{4} \theta^{\prime} F_{1}-\frac{1}{2} f^{\prime} \Theta_{l}=\frac{1}{2} \sqrt{(1+\operatorname{Pr})} \Theta_{0}, \\
& \frac{1+\operatorname{Pr}}{\mathrm{Sc}} \Phi_{1}^{\prime \prime}+\frac{3}{4} f \Phi_{1}^{\prime}+\frac{5}{4} \varphi^{\prime} F_{1}-\frac{1}{2} f^{\prime} \Phi_{1}=\frac{1}{2} \sqrt{1+\operatorname{Pr}} \Phi_{0} .
\end{aligned}
$$

For $m \geq 2$

$$
\begin{aligned}
& (1+\operatorname{Pr}) F_{m}^{\prime \prime \prime}+\frac{3}{4} f F_{m}^{\prime \prime}+\left(\frac{3}{4}+\frac{m}{2}\right) f^{\prime \prime} F_{m}-\left(1+\frac{m}{2}\right) f^{\prime} F_{m}^{\prime}+ \\
& +(1-\omega)(1+\operatorname{Pr}) \operatorname{Ri}_{m}+\omega(1+\operatorname{Pr}) \operatorname{Ri} \Phi_{m}=\frac{1}{2} \sqrt{1+\operatorname{Pr}} F_{m-1}^{\prime}, \\
& \frac{(1+\operatorname{Pr})}{\operatorname{Pr}}\left\{1+\frac{4}{3} R_{d}(1+\Delta \theta)^{3}\right\} \Theta_{m}^{\prime \prime}+\frac{8(1+\operatorname{Pr}) \Delta}{\operatorname{Pr}} R_{d}(1+\Delta \theta)^{2} \theta^{\prime} \Theta_{m}^{\prime}+ \\
& +\frac{8(1+\operatorname{Pr}) \Delta^{2}}{\operatorname{Pr}} R_{d}(1+\Delta \theta) \theta^{\prime 2} \Theta_{m}+\frac{4(1+\operatorname{Pr}) \Delta}{\operatorname{Pr}} R_{d}(1+\Delta \theta)^{2} \theta^{\prime \prime} \Theta_{m}+ \\
& +\frac{3}{4} f \Theta_{m}^{\prime}+\left(\frac{3}{4}+\frac{m}{2}\right) \theta^{\prime} F_{m}-\frac{m}{2} f^{\prime} \Theta_{m}=\frac{1}{2} \sqrt{(1+\operatorname{Pr})} \Theta_{m-1}, \\
& \frac{1+\operatorname{Pr}}{\mathrm{Sc}} \Phi_{m}^{\prime \prime}+\frac{3}{4} f \Phi_{m}^{\prime}+\left(\frac{3}{4}+\frac{m}{2}\right) \varphi^{\prime} F_{m}-\frac{1}{2} f^{\prime} \Phi_{m}=\frac{1}{2} \sqrt{1+\operatorname{Pr}} \Phi_{m-1}
\end{aligned}
$$

The corresponding boundary conditions are

$$
\begin{aligned}
& F_{m}(0)=F_{m}^{\prime}(0)=\Theta_{m}(0)=\Phi_{m}(0)=0, \Theta_{0}(0)=1, \Phi_{0}(0)=1 . \text { for } m=0,1,2, \ldots \\
& F_{0}^{\prime}(\infty)=(1+\operatorname{Pr})^{1 / 2}, F_{m}^{\prime}(\infty)=0, \Theta_{m}(\infty)=0, \Phi_{m}(\infty)=0 \quad \text { for } \quad m=0,1,2, \ldots
\end{aligned}
$$


Here primes denote derivative with respect to $\eta$. Since Eqs (3.2)-(3.10) are linear and coupled, we solve these using the implicit Runge-Kutta-Butcher [17] initial value solver together with NachtsheimSwigert [18] iteration up to $O\left(\xi^{10}\right)$.

\subsection{Asymptotic solutions for large $\xi$}

We now concentrate on the solutions of Eqs (2.26)-(2.28) as $\xi$ tends to infinity. Under this circumstance, the boundary layer characteristics may vary within a limited region adjacent to the surface. Accordingly, we seek series solutions using the following transformations

$$
F(\xi, \eta)=\xi^{-1 / 2} G(\xi, Y), \Theta(\xi, \eta)=\Theta(\xi, Y), \Phi(\xi, \eta)=\Phi(\xi, Y), \quad Y=\xi^{1 / 2} \eta
$$

We thus obtain

$$
\begin{aligned}
& (1+\operatorname{Pr}) \frac{\partial^{3} G}{\partial Y^{3}}+\frac{3}{4} \xi^{-1 / 2} f \frac{\partial^{2} G}{\partial Y^{2}}+\xi^{-1}\left\{1-f^{\prime} \frac{\partial G}{\partial Y}\right\}+\frac{1}{2} \xi^{-3 / 2} f^{\prime \prime} G+ \\
& +i\left\{(1+\operatorname{Pr})-\sqrt{1+\operatorname{Pr}} \frac{\partial G}{\partial Y}\right\}+\operatorname{Ri}(1-\omega)(1+\operatorname{Pr}) \xi^{-1} \Theta+ \\
& +\operatorname{Ri} \omega(1+\operatorname{Pr}) \xi^{-1} \Phi=\frac{1}{2}\left\{f^{\prime}\left(\frac{\partial^{2} G}{\partial Y \partial \xi}+\frac{Y}{2 \xi} \frac{\partial^{2} G}{\partial Y^{2}}\right)-\xi^{-1 / 2} f^{\prime \prime}\left(\frac{\partial G}{\partial \xi}+\frac{Y}{2 \xi} \frac{\partial G}{\partial Y}\right)\right\}, \\
& \frac{1+\operatorname{Pr}}{\operatorname{Pr}} \frac{\partial^{2} \Theta}{\partial Y^{2}}+\frac{3}{4} \xi^{-1 / 2} f^{\prime} \frac{\partial \Theta}{\partial Y}+\frac{1}{2} \xi^{-3 / 2} \theta^{\prime} G-i \sqrt{1+\operatorname{Pr} \Theta=} \\
& =\frac{1}{2}\left\{f^{\prime}\left(\frac{\partial \Theta}{\partial \xi}+\frac{Y}{2 \xi} \frac{\partial \Theta}{\partial Y}\right)-\xi^{-1 / 2} \theta^{\prime}\left(\frac{\partial G}{\partial \xi}+\frac{Y}{2 \xi} \frac{\partial G}{\partial Y}\right)\right\}, \\
& \frac{1+\operatorname{Pr}}{\operatorname{Sc}} \frac{\partial^{2} \Phi}{\partial Y^{2}}+\frac{3}{4} \xi^{-1 / 2} f \frac{\partial \Phi}{\partial Y}+\frac{1}{2} \xi^{-3 / 2} \varphi^{\prime} G-i \sqrt{1+\operatorname{Pr}} \Phi= \\
& =\frac{1}{2}\left\{f^{\prime}\left(\frac{\partial \Phi}{\partial \xi}+\frac{Y}{2 \xi} \frac{\partial \Phi}{\partial Y}\right)-\xi^{-1 / 2} \varphi^{\prime}\left(\frac{\partial G}{\partial \xi}+\frac{Y}{2 \xi} \frac{\partial G}{\partial Y}\right)\right\} .
\end{aligned}
$$

For small values of $\eta$, we expand the solutions of Eqs (2.15)-(2.17) in the power series

$$
\begin{aligned}
& f=a_{2} \eta^{2}+a_{3} \eta^{3}+a_{4} \eta^{4}+a_{5} \eta^{5}+\cdots, \\
& \theta=b_{0}+b_{1} \eta+b_{2} \eta^{2}+b_{3} \eta^{3}+b_{4} \eta^{4}+b_{5} \eta^{5}+\cdots, \\
& \varphi=c_{0}+c_{1} \eta+c_{2} \eta^{2}+c_{3} \eta^{3}+c_{4} \eta^{4}+c_{5} \eta^{5}+\cdots
\end{aligned}
$$

Using Eqs (3.17)-(3.19) in Eqs (2.15)-(2.19) yields

$$
a_{2}=\frac{1}{2} f^{\prime \prime}(0), \quad b_{0}=\theta(0), \quad b_{1}=\theta^{\prime}(0), \cdots . c_{0}=\varphi(0), \quad c_{1}=\varphi^{\prime}(0), \ldots .
$$


Now, the solutions of Eqs (3.14)-(3.16) can be obtained in the following forms

$$
G(\xi, Y)=\sum_{m=0}^{\infty} \xi^{-m / 2} E_{m}(Y), \quad \Theta(\xi, Y)=\sum_{m=0}^{\infty} \xi^{-m / 2} L_{m}(Y)
$$

and

$$
\Phi(\xi, Y)=\sum_{m=0}^{\infty} \xi^{-m / 2} N_{m}(Y)
$$

Substituting Eqs (3.21) into Eqs (3.14)-(3.16), and equating the like powers of $\xi$, we obtain

$$
\begin{aligned}
& E_{0}^{\prime \prime \prime}-\frac{i}{\sqrt{1+\operatorname{Pr}}} E_{0}^{\prime}=-i \\
& E_{1}^{\prime \prime \prime}-\frac{i}{\sqrt{1+\operatorname{Pr}}} E_{1}^{\prime}=0 \\
& E_{2}^{\prime \prime \prime}-\frac{i}{\sqrt{1+\operatorname{Pr}}} E_{2}^{\prime}=-\left\{1+(1-w) \operatorname{Ri} L_{0}+w \operatorname{Ri} N_{0}\right\}, \\
& E_{3}^{\prime \prime \prime}-\frac{i}{\sqrt{1+\operatorname{Pr}}} E_{3}^{\prime}=-\frac{a_{2}}{4(1+\operatorname{Pr})}\left(Y^{2} E_{0}^{\prime \prime}-6 Y E_{0}^{\prime}+4 E_{0}\right)-(1-w) \operatorname{Ri} L_{1}-w \operatorname{Ri} N_{1}, \\
& \left\{1+\frac{4}{3} R_{d}(1+\Delta \theta)^{3}\right\} L_{0}^{\prime \prime}-\frac{i \operatorname{Pr}}{\sqrt{1+\operatorname{Pr}}} L_{0}=0 \\
& \left\{1+\frac{4}{3} R_{d}(1+\Delta \theta)^{3}\right\} L_{l}^{\prime \prime}-\frac{i \operatorname{Pr}}{\sqrt{1+\operatorname{Pr}}} L_{1}=-4 \Delta R_{d}(1+\Delta)^{2} b_{1}\left(Y L_{0}^{\prime \prime}+2 L_{0}^{\prime}\right), \\
& \left\{1+\frac{4}{3} R_{d}(1+\Delta \theta)^{3}\right\} L_{2}^{\prime \prime}-\frac{i \operatorname{Pr}}{\sqrt{1+\operatorname{Pr}}} L_{2}=-4 \Delta R_{d}(1+\Delta)^{2} b_{1}\left(Y L_{1}^{\prime \prime}+2 L_{1}^{\prime}\right)+ \\
& -4 \Delta R_{d}(1+\Delta)\left[(1+\Delta) b_{2}+\Delta b_{1}{ }^{2}\right] \times\left(Y^{2} L_{0}^{\prime \prime}+4 Y L_{0}{ }^{\prime}+2 L_{0}\right), \\
& \left\{1+\frac{4}{3} R_{d}(1+\Delta \theta)^{3}\right\} L_{3}^{\prime \prime}-\frac{i \operatorname{Pr}}{\sqrt{1+\operatorname{Pr}}} L_{3}=-4 \Delta R_{d}(1+\Delta)^{2} b_{1}\left(Y L_{2}^{\prime \prime}+2 L_{2}^{\prime}\right)+ \\
& -4 \Delta R_{d}(1+\Delta)\left[(1+\Delta) b_{2}+\Delta b_{1}^{2}\right] \times\left(Y^{2} L_{l}^{\prime \prime}+4 Y L_{1}^{\prime}+2 L_{1}\right)+ \\
& -\frac{4 \Delta}{3} R_{d}\left[3(1+\Delta)^{2} b_{3}+6 \Delta(1+\Delta) b_{1} b_{2}+\Delta^{2} b_{1}^{3}\right] \times\left(Y^{3} L_{0}^{\prime \prime}+6 Y^{2} L_{0}{ }^{\prime}+6 Y L_{0}\right)+ \\
& -\frac{\operatorname{Pr}}{4(1+\operatorname{Pr})}\left\{a_{2} Y^{2} L_{0}^{\prime}+b_{1}\left(Y E_{0}^{\prime}+2 E_{0}\right)\right\}
\end{aligned}
$$

and 


$$
\begin{aligned}
& N_{0}^{\prime \prime}-\frac{i \mathrm{Sc}}{\sqrt{1+\operatorname{Pr}}} N_{0}=0, \\
& N_{1}^{\prime \prime}-\frac{i \mathrm{Sc}}{\sqrt{1+\operatorname{Pr}}} N_{1}=0, \\
& N_{2}^{\prime \prime}-\frac{i \mathrm{Sc}}{\sqrt{1+\operatorname{Pr}}} N_{2}=0, \\
& N_{3}^{\prime \prime}-\frac{i \mathrm{Sc}}{\sqrt{1+\operatorname{Pr}}} N_{3}=-\frac{\mathrm{Sc}}{4(1+\operatorname{Pr})}\left\{a_{2} Y^{2} N_{0}^{\prime}+c_{1}\left(Y E_{0}^{\prime}+2 E_{0}\right)\right\} .
\end{aligned}
$$

The associated boundary conditions are

$$
\begin{aligned}
& E_{m}(0)=E_{m}^{\prime}(0)=0, \quad E_{0}^{\prime}(\infty)=(1+\operatorname{Pr})^{1 / 2}, \quad E_{m}^{\prime}(\infty)=0, \quad \text { for } m=0,1, \ldots, \\
& L_{0}(0)=1, \quad L_{m}(0)=L_{m}(\infty)=0, \quad \text { for } \quad m=1,2, \ldots, \\
& N_{0}(0)=1, \quad N_{m}(0)=N_{m}(\infty)=0, \quad \text { for } \quad m=1,2, \ldots
\end{aligned}
$$

Solving the above equations, we can find the expressions for $F^{\prime \prime}(\xi, 0), \Theta^{\prime}(\xi, 0)$ and $\Phi^{\prime}(\xi, 0)$ as

$$
\begin{aligned}
& (1+\operatorname{Pr})^{1 / 4} \xi^{-1 / 2} F^{\prime \prime}(\xi, 0)=\sum_{m=0}^{\infty} \xi^{-m / 2} E_{m}^{\prime \prime}(0) \\
& (1+\operatorname{Pr})^{-1 / 4} \xi^{-1 / 2} \Theta^{\prime}(\xi, 0)=\sum_{m=0}^{\infty} \xi^{-m / 2} L_{m}{ }^{\prime}(0), \\
& (1+\operatorname{Pr})^{-1 / 4} \xi^{-1 / 2} \Phi^{\prime}(\xi, 0)=\sum_{m=0}^{\infty} \xi^{-m / 2} N_{m}{ }^{\prime}(0),
\end{aligned}
$$

where

$$
\begin{aligned}
& E_{0}^{\prime \prime}(0)=(1+\operatorname{Pr})^{1 / 4} r, \quad E_{1}^{\prime \prime}(0)=0, \quad E_{2}^{\prime \prime}(0)=\frac{(1+\operatorname{Pr})^{1 / 4}\left(G_{0}+G_{1}\right)}{(\sqrt{A}+\sqrt{\operatorname{Pr}})(1+\sqrt{\mathrm{Sc}}) r^{2}}, \\
& E_{3}^{\prime \prime}(0)=\frac{G_{2}-G_{3}}{16(\sqrt{A}+\sqrt{\operatorname{Pr}})^{3} r^{2}},
\end{aligned}
$$




$$
\begin{aligned}
& L_{0}^{\prime}(0)=-\frac{r \sqrt{\operatorname{Pr}}}{\sqrt{A}(1+\operatorname{Pr})^{1 / 4}}, \quad L_{1}^{\prime}(0)=-\frac{3 B}{4 A}, \quad L_{2}^{\prime}(0)=\frac{\left(-3 B^{2}+8 A G_{4}\right)(1+\operatorname{Pr})^{1 / 4}}{32 \sqrt{\operatorname{Pr}} A^{3 / 2} r}, \\
& L_{3}^{\prime}(0)=\frac{\left(3 B^{3}-12 A B G_{4}+24 A^{2} G_{5}\right) \sqrt{1+\operatorname{Pr}}}{64 \operatorname{Pr} A^{2} r^{2}}-\frac{G_{6}}{16 \sqrt{1+\operatorname{Pr}}(\sqrt{A}+\sqrt{\operatorname{Pr}})^{2} r^{2}}, \\
& N_{0}^{\prime}(0)=-\frac{r \sqrt{\mathrm{Sc}}}{(1+\operatorname{Pr})^{1 / 4}}, \quad N_{1}^{\prime}(0)=0, N_{2}^{\prime}(0)=0, \quad N_{3}^{\prime}(0)=-\frac{G_{7}+G_{8}}{16 r^{2}(1+\sqrt{\mathrm{Sc}})^{2} \sqrt{1+\operatorname{Pr}}},
\end{aligned}
$$

with

$$
\begin{aligned}
& r=\sqrt{i}, \quad A=\left[1+\frac{4}{3} R_{d}(1+\Delta \theta)^{3}\right], B=4 \Delta R_{d}(1+\Delta)^{2} b_{1}, \\
& G_{0}=\sqrt{A}[1+\sqrt{\mathrm{Sc}}+\operatorname{Ri}\{1-\sqrt{\mathrm{Sc}}(-1+\omega)\}], \quad G_{1}=\sqrt{\operatorname{Pr}}(1+\sqrt{\mathrm{Sc}}+\operatorname{Ri} \omega), \\
& G_{2}=4 B(3 \sqrt{A}+\sqrt{\operatorname{Pr}}) \sqrt{1+\operatorname{Pr}} \operatorname{Ri}(-1+\omega), G_{3}=9 a_{2}(\sqrt{A}+\sqrt{\operatorname{Pr}})^{3}, \\
& G_{4}=4 \Delta R_{d}(1+\Delta)\left[(1+\Delta) b_{2}+\Delta b_{1}^{2}\right], G_{5}=\frac{4}{3} \Delta R_{d}\left[3(1+\Delta)^{2} b_{3}+6 \Delta(1+\Delta) b_{1} b_{2}+\Delta b_{1}^{3}\right], \\
& G_{6}=\left[a_{2} \operatorname{Pr}+A\left(a_{2}-12 B \sqrt{1+\operatorname{Pr}}\right)+2 \sqrt{A} \sqrt{\operatorname{Pr}}\left(a_{2}-8 b_{1} \sqrt{1+\operatorname{Pr}}\right)\right], G_{7}=a_{2}(1+\sqrt{\mathrm{Sc}})^{2}, \\
& G_{8}=4 c_{1}(3+4 \sqrt{\mathrm{Sc}}) \sqrt{1+\operatorname{Pr}} .
\end{aligned}
$$

\section{Results and discussion}

In this study, we have analyzed the problem of an unsteady laminar mixed convection boundary layer flow of viscous incompressible fluid near a vertical wedge surface. The governing equations have been solved by two distinct methods, namely, the straightforward finite difference method for the entire frequency range, and the extended series solution for the low frequency range and the asymptotic series expansion method for the high frequency range. It is worth mentioning that the effect of the constant coefficients $(1+$ $\operatorname{Pr})^{1 / 4},(1+\operatorname{Pr})^{-1 / 4}$ and $(1+\operatorname{Pr})^{-1 / 4}$ within the definitions of the skin friction, heat transfer and mass transfer (Eqs (3.22)-(3.24)), respectively, has not been included into the results. 

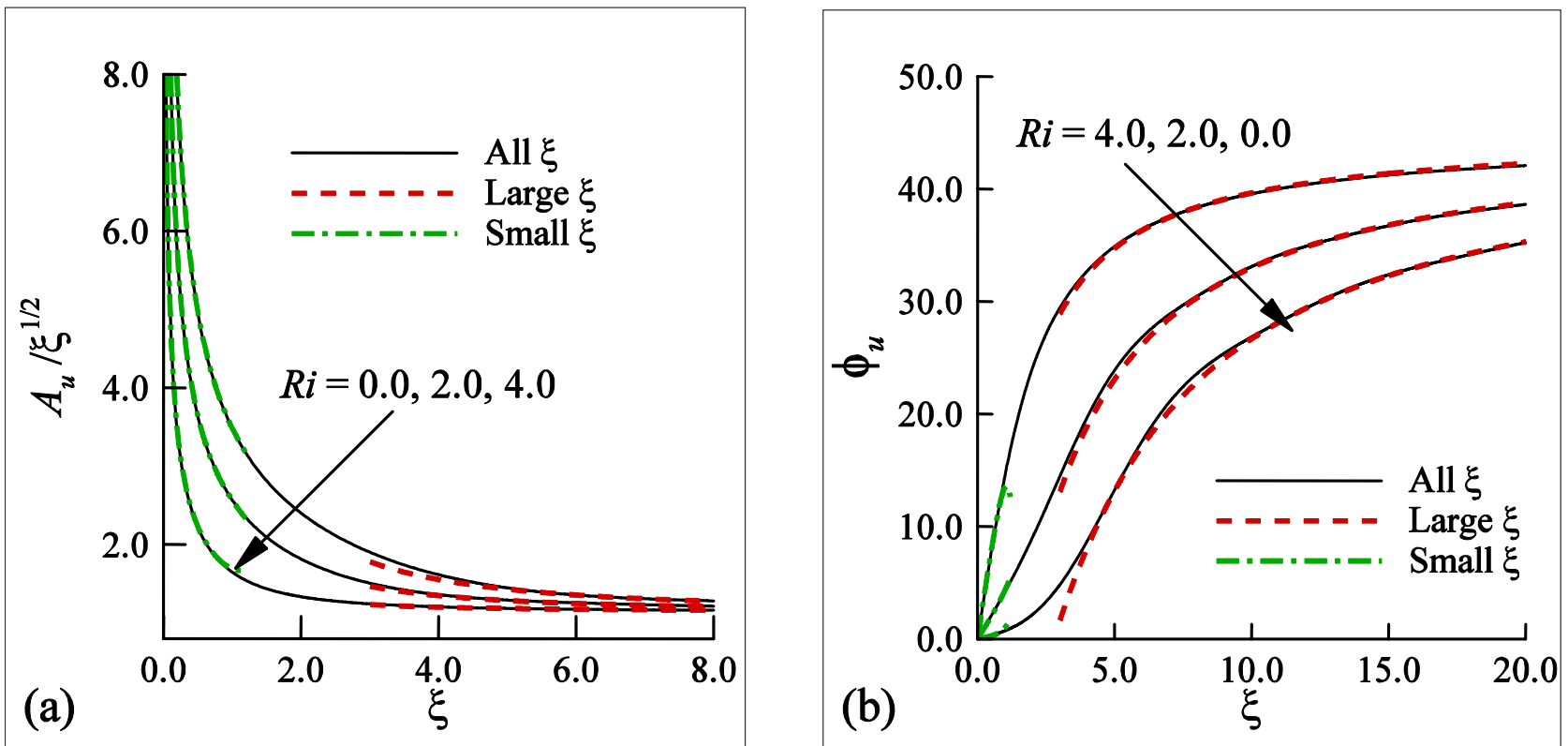

Fig.1. (a) Amplitude and (b) phase angle of skin friction for different values of $\operatorname{Ri}$ while $\operatorname{Pr}=0.72, w=0.5$, $\mathrm{Sc}=0.22, R_{d}=0.5, \Delta=0.1$.

With the aim of validating the numerical solutions, a comparison of the amplitudes and phase angles of skin friction obtained by the numerical solutions for all $\xi$ and the series solutions for small and large $\xi$ are shown in Fig.1. It is obvious from the figures that the solutions are in excellent agreement. Besides Fig.1 depicts the effects of varying the effects of Richardson's number, $\mathrm{Ri}$, on the amplitudes and phase angles of the skin friction. It is seen that the amplitudes of skin friction are higher for increased Richardson's number, $\mathrm{Ri}$, while the phase angles are lower. As the Richardson's number, Ri, increases, mixed convection of flow, heat transfer and mass transfer increases. Accordingly, the amplitudes of skin friction are higher for higher Ri. But the rate of change of skin friction from the leading edge to the downstream region is higher for lower $\mathrm{Ri}$ so that the phase angles of skin friction are higher for lower Ri.
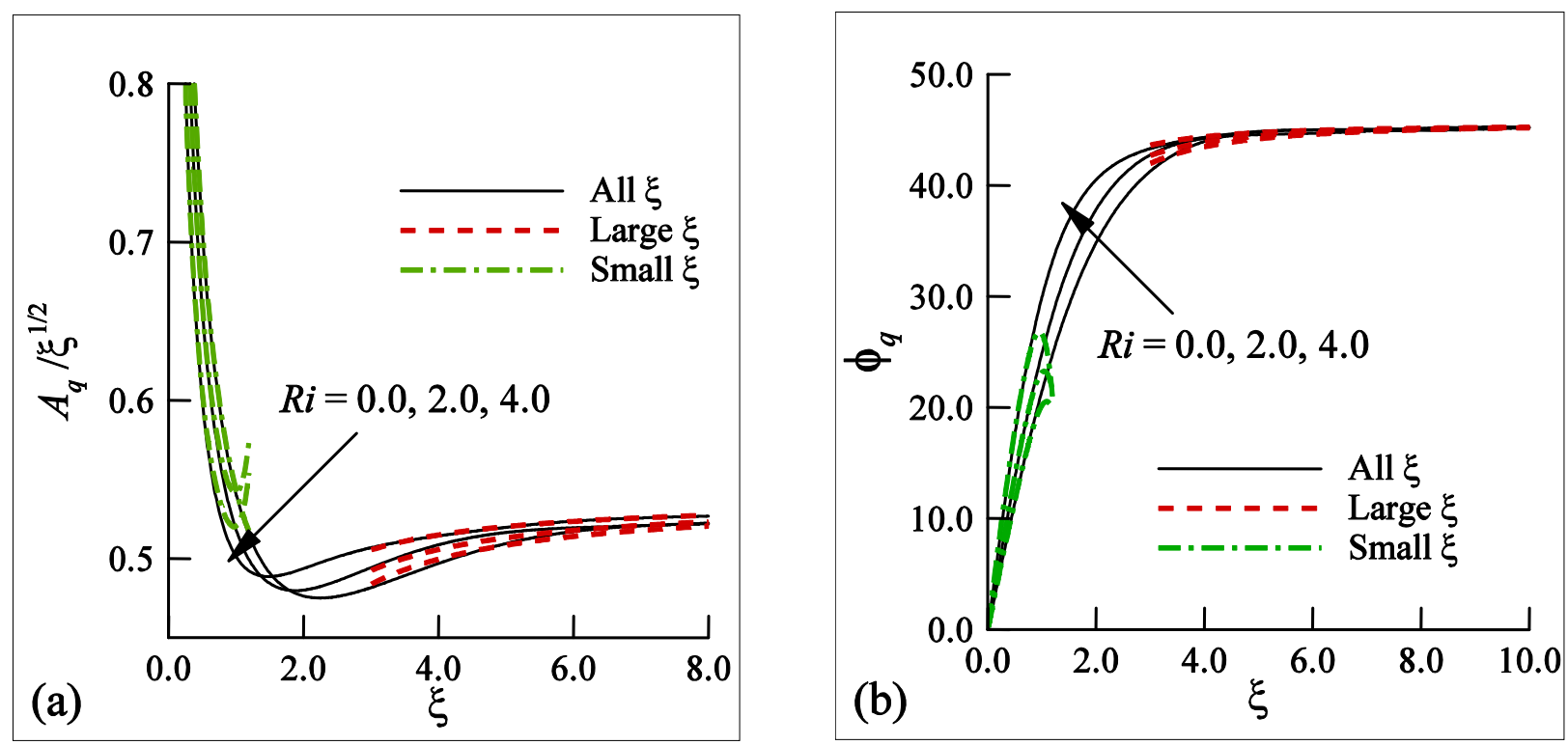

Fig.2. (a) Amplitude and (b) phase angle of heat transfer for different values of $\operatorname{Ri}$ while $\operatorname{Pr}=0.72, w=0.5$, $\mathrm{Sc}=0.22, R_{d}=0.5, \Delta=0.1$. 
The effects of varying the Richardson's number, Ri, on (a) amplitudes and (b) phase angles of heat transfer are shown in Fig.2. With an increase of Ri, the amplitudes of heat transfer are higher near the leading edge while the reverse case is observed in the downstream region. On the other hand, for smaller $\mathrm{Ri}$ the phase angles are higher near the leading edge and then they become lower in the downstream region.
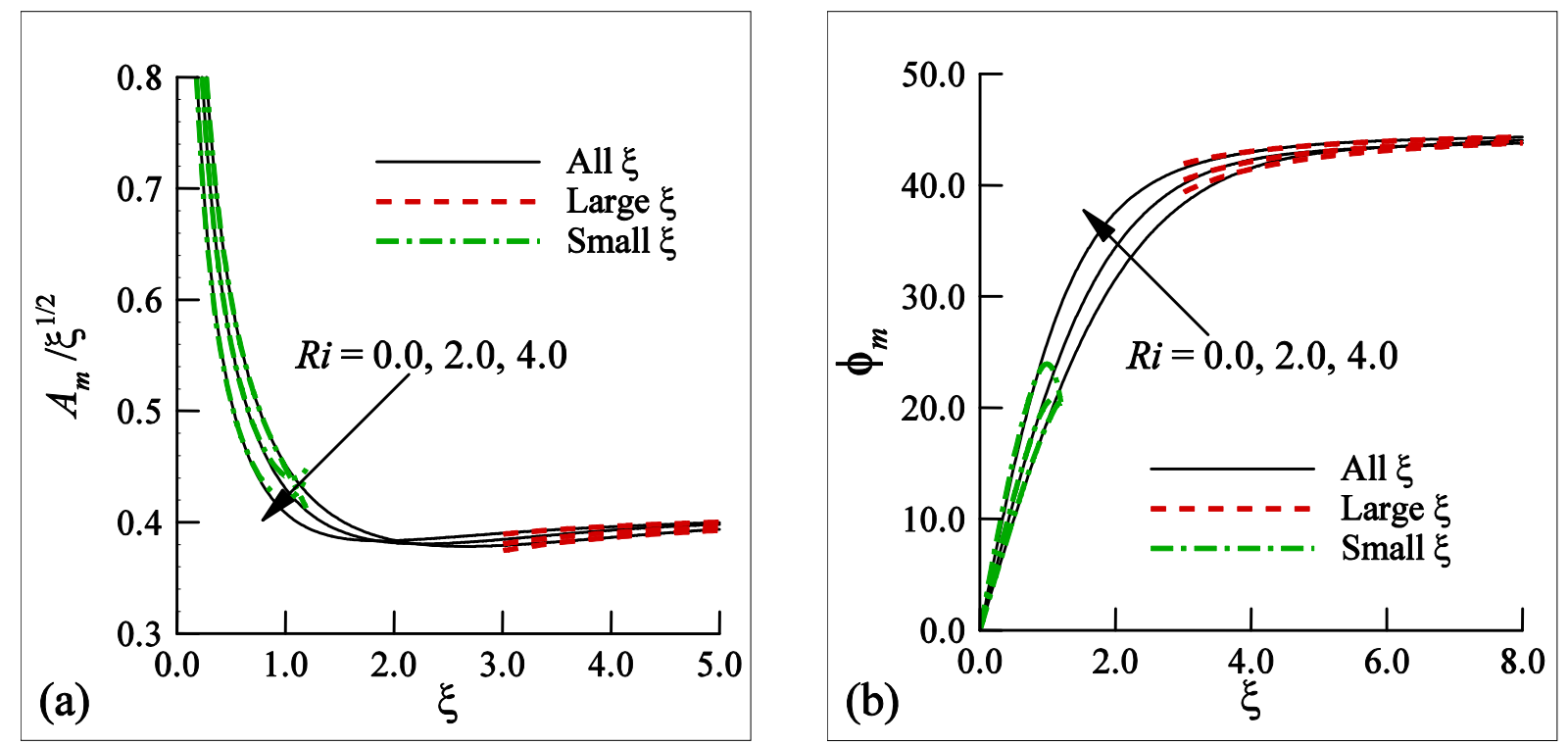

Fig.3. (a) Amplitude and (b) phase angle of mass transfer for different values of Ri while $\operatorname{Pr}=0.72$, $w=0.5, \mathrm{Sc}=0.22, R_{d}=0.5, \Delta=0.1$.

Figure 3 exhibits the effects of varying the Richardson's number, Ri, on the amplitudes and phase angles of mass transfer. The amplitudes of mass transfer are higher near the leading edge with an increase of $\mathrm{Ri}$, while the reverse case is observed in the downstream region. Nevertheless for smaller Ri the phase angles are higher. Finally, the amplitudes and phase angles tend to an asymptotic value for all Ri.
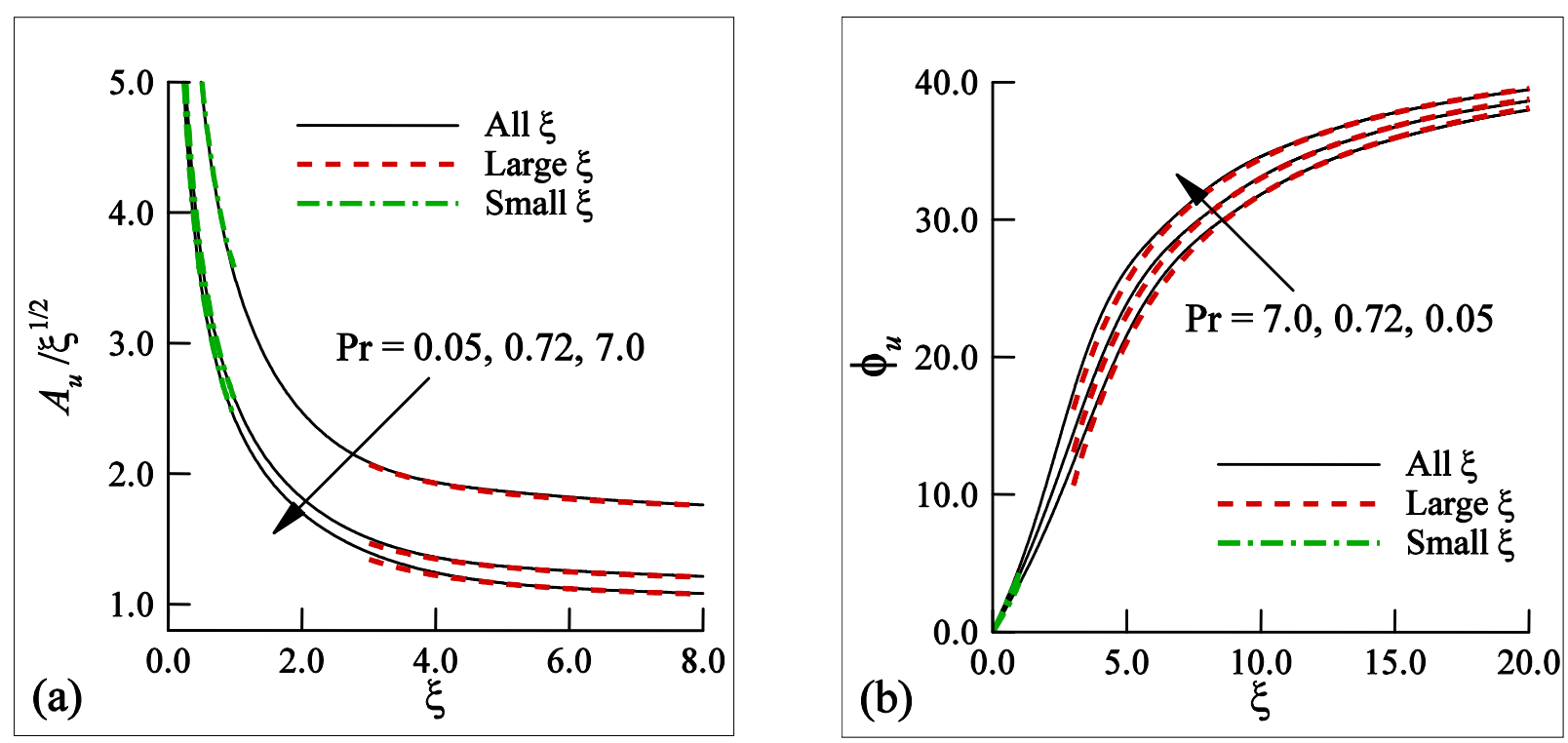

Fig.4. (a) Amplitude and (b) phase angle of skin friction for different values of Pr while $\mathrm{Ri}=2.0, w=0.5$, $\mathrm{Sc}=0.22, R_{d}=0.5, \Delta=0.5$. 
The influences of the Prandtl number, Pr, on the amplitudes and phase angles of the skin friction are illustrated in Fig.4. From this figure and Tab.1, it is observed that when the Prandtl number is increased, the amplitudes and phase angles of skin friction increase. This is for the reason that the Prandtl number becomes high owing to either increase of the kinematic viscosity or decrease of the thermal diffusivity of the fluid, and the increase of the skin friction is the result of this change of the fluid property.

Table1. Amplitude and phase angle of skin friction for different $\operatorname{Pr}$ while $\mathrm{Ri}=2.0$.

\begin{tabular}{|c|c|c|c|c|}
\hline \multirow[t]{2}{*}{$\xi$} & \multicolumn{2}{|r|}{$A_{u}$} & \multicolumn{2}{|r|}{$\phi_{u}$} \\
\hline & All $\xi$ & Small \& Large $\xi$ & All $\xi$ & Small and Large $\xi$ \\
\hline \multicolumn{5}{|c|}{$\operatorname{Pr}=0.05$} \\
\hline 0.01 & 24.21504 & $24.39176^{s}$ & 0.03090 & $0.03135^{s}$ \\
\hline 0.10 & 7.65732 & $7.71290^{s}$ & 0.34034 & $0.31396^{s}$ \\
\hline 0.50 & 3.42191 & $3.44411^{s}$ & 1.70988 & $1.61944^{s}$ \\
\hline 1.00 & 2.41451 & $2.42084^{s}$ & 3.49818 & $3.63779^{s}$ \\
\hline 2.00 & 1.70030 & $1.41458^{s}$ & 7.55133 & $9.56755^{s}$ \\
\hline 3.00 & 1.39702 & $1.34327^{l}$ & 12.28315 & $10.68621^{l}$ \\
\hline 4.00 & 1.24198 & $1.22190^{l}$ & 17.20686 & $16.68792^{l}$ \\
\hline 6.00 & 1.12263 & $1.11855^{l}$ & 24.95134 & $24.36954^{l}$ \\
\hline 8.00 & 1.08296 & $1.07706^{l}$ & 29.14756 & $28.87964^{l}$ \\
\hline 10.00 & 1.05770 & $1.05622^{l}$ & 31.82646 & $31.80292^{l}$ \\
\hline \multicolumn{5}{|c|}{$\operatorname{Pr}=0.72$} \\
\hline 0.01 & 25.73804 & $24.23397^{s}$ & 0.03861 & $0.04543^{S}$ \\
\hline 0.10 & 8.13899 & $7.66298^{s}$ & 0.40594 & $0.45460^{s}$ \\
\hline 0.50 & 3.63751 & $3.42219^{s}$ & 2.02972 & $2.30713^{s}$ \\
\hline 1.00 & 2.56769 & $2.41203^{s}$ & 4.15585 & $4.81258^{s}$ \\
\hline 2.00 & 1.81466 & $1.48212^{s}$ & 8.97085 & $11.80142^{s}$ \\
\hline 3.00 & 1.50619 & $1.46646^{l}$ & 14.41984 & $13.10832^{l}$ \\
\hline 4.00 & 1.35994 & $1.34785^{l}$ & 19.67463 & $18.90033^{l}$ \\
\hline 6.00 & 1.25663 & $1.24767^{l}$ & 26.80379 & $26.14150^{l}$ \\
\hline 8.00 & 1.21358 & $1.20768^{l}$ & 30.45161 & $30.32083^{l}$ \\
\hline 10.00 & 1.18805 & $1.18762^{l}$ & 33.10317 & $33.00724^{l}$ \\
\hline \multicolumn{5}{|c|}{$\operatorname{Pr}=7.0$} \\
\hline 0.01 & 35.19503 & $32.39354^{S}$ & 0.04477 & $0.00830^{s}$ \\
\hline 0.10 & 11.12919 & $10.24231^{s}$ & 0.45215 & $0.08319^{s}$ \\
\hline 0.50 & 4.96918 & $4.56520^{s}$ & 2.26401 & $0.44264^{s}$ \\
\hline 1.00 & 3.49952 & $3.19606^{s}$ & 4.73482 & $1.04939^{s}$ \\
\hline 2.00 & 2.47273 & $2.88774^{s}$ & 10.73652 & $13.31676^{s}$ \\
\hline 3.00 & 2.08757 & $2.07070^{l}$ & 17.39014 & $16.22144^{l}$ \\
\hline 4.00 & 1.93413 & $1.92644^{l}$ & 22.84133 & $21.65324^{l}$ \\
\hline 6.00 & 1.82116 & $1.80549^{l}$ & 28.73856 & $28.27460^{l}$ \\
\hline 8.00 & 1.76074 & $1.75736^{l}$ & 32.23035 & $32.02920^{l}$ \\
\hline 10.00 & 1.73675 & $1.73323^{l}$ & 34.59221 & $34.42276^{l}$ \\
\hline
\end{tabular}

Notes: Here and hereafter, ${ }^{s}$ and ${ }^{l}$ represent the solutions due to series solution for small $\xi$ and asymptotic solution for large $\xi$, respectively. 

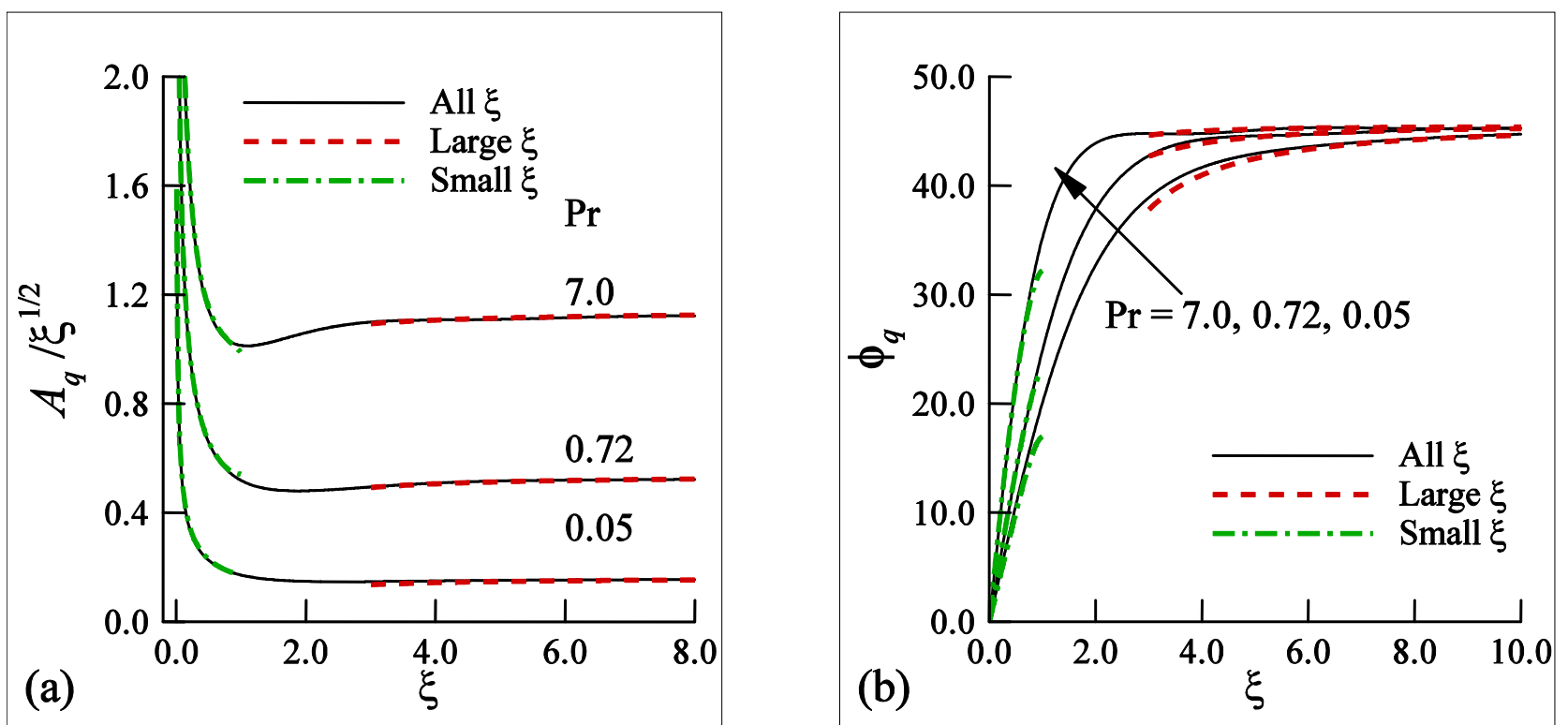

Fig.5. (a) Amplitude and (b) phase angle of heat transfer for different values of Pr while $\mathrm{Ri}=2.0, w=0.5$, $\mathrm{Sc}=0.22, R_{d}=0.5, \Delta=0.5$.
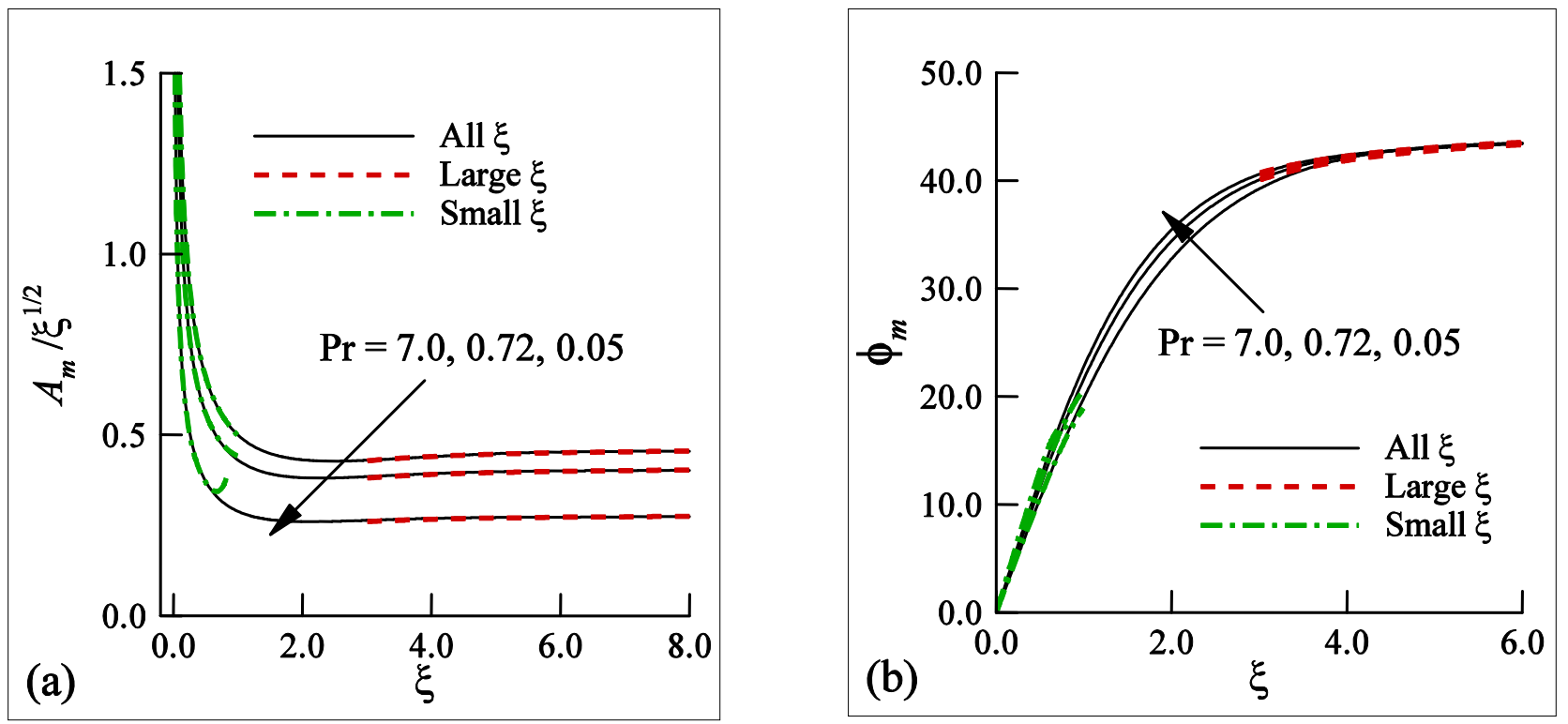

Fig.6. (a) Amplitude and (b) phase angle of mass transfer for different values of $\operatorname{Pr}$ while $\mathrm{Ri}=2.0, w=0.5$, $\mathrm{Sc}=0.22, R_{d}=0.5, \Delta=0.5$.

Figure 5 presents a comparison between the numerical solutions for all $\xi$ and the series solutions for small and large $\xi$ in terms of the amplitudes and phase angles of heat transfer. Evidently, the solutions obtained by the numerical solutions provide a good agreement with the series solutions. Also the effects of the Prandtl number on the heat transfer are comprehensible from Fig.5. It is found that the increment of the Prandtl number causes an increase of the amplitudes and phase angles of heat transfer near the leading edge. Since the Prandtl number increases due to either an increase of the kinematic viscosity or a decrease of the thermal diffusivity of the fluid, hence heat is accumulated near the leading edge that results in the increase of the amplitudes and phase angles of heat transfer. 
Figure 6 illustrates the effects of changing the Prandtl number, Pr, on the amplitudes and phase angles of mass transfer. It is seen from the figure that the amplitudes of mass transfer reduce and the phase angles of mass transfer raise with increasing the Prandtl number, Pr. This is due to the fact that the Prandtl number becomes high in consequence of either an increase of the kinematic viscosity or decrease of the thermal diffusivity of the fluid, and the increase of the skin friction is the result of this change in the fluid property.
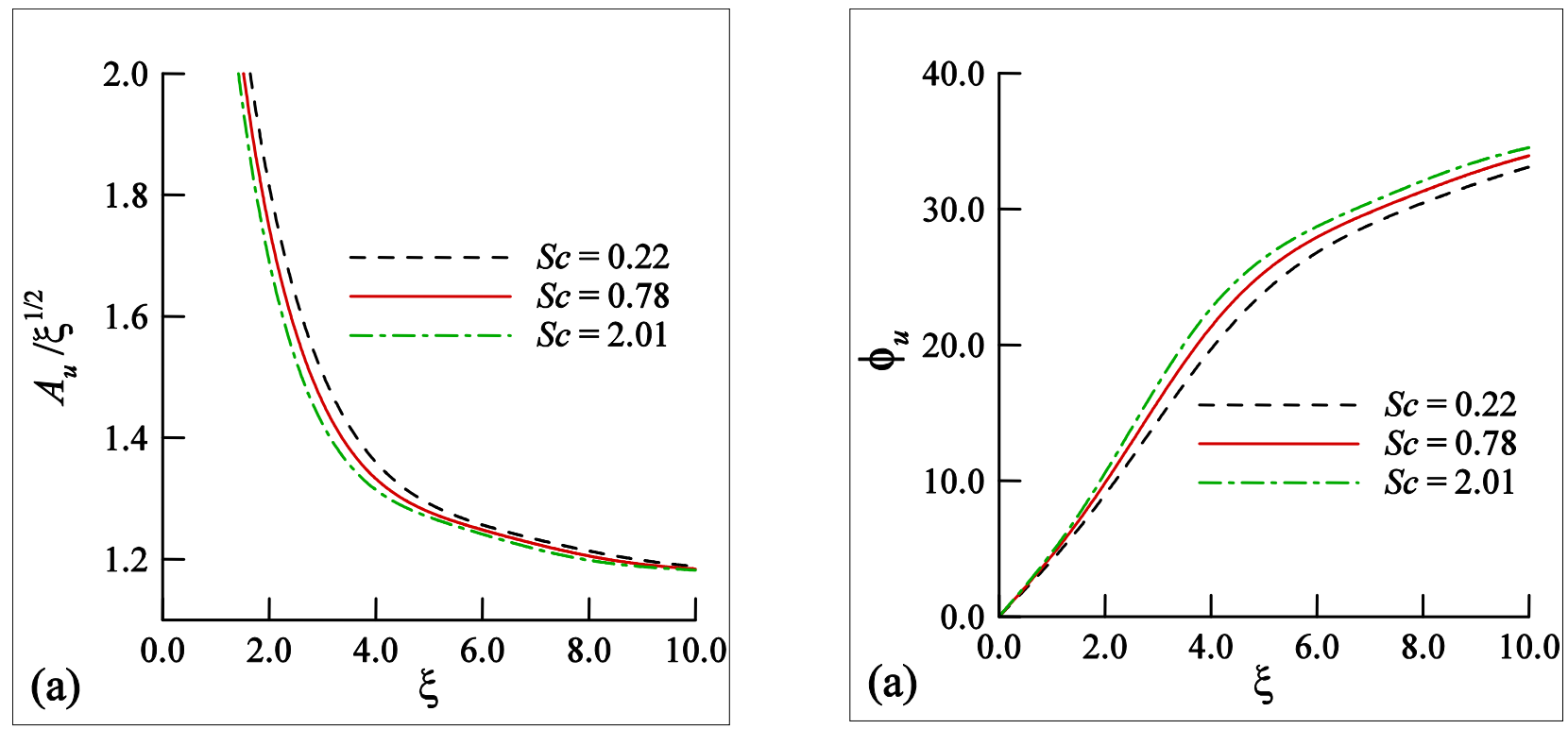

Fig.7. (a) Amplitude and (b) phase angle of skin friction for different values of $\mathrm{Sc}$ while $\mathrm{Pr}=0.72, \mathrm{Ri}=2.0$, $w=0.5, R_{d}=0.5, \Delta=0.5$.
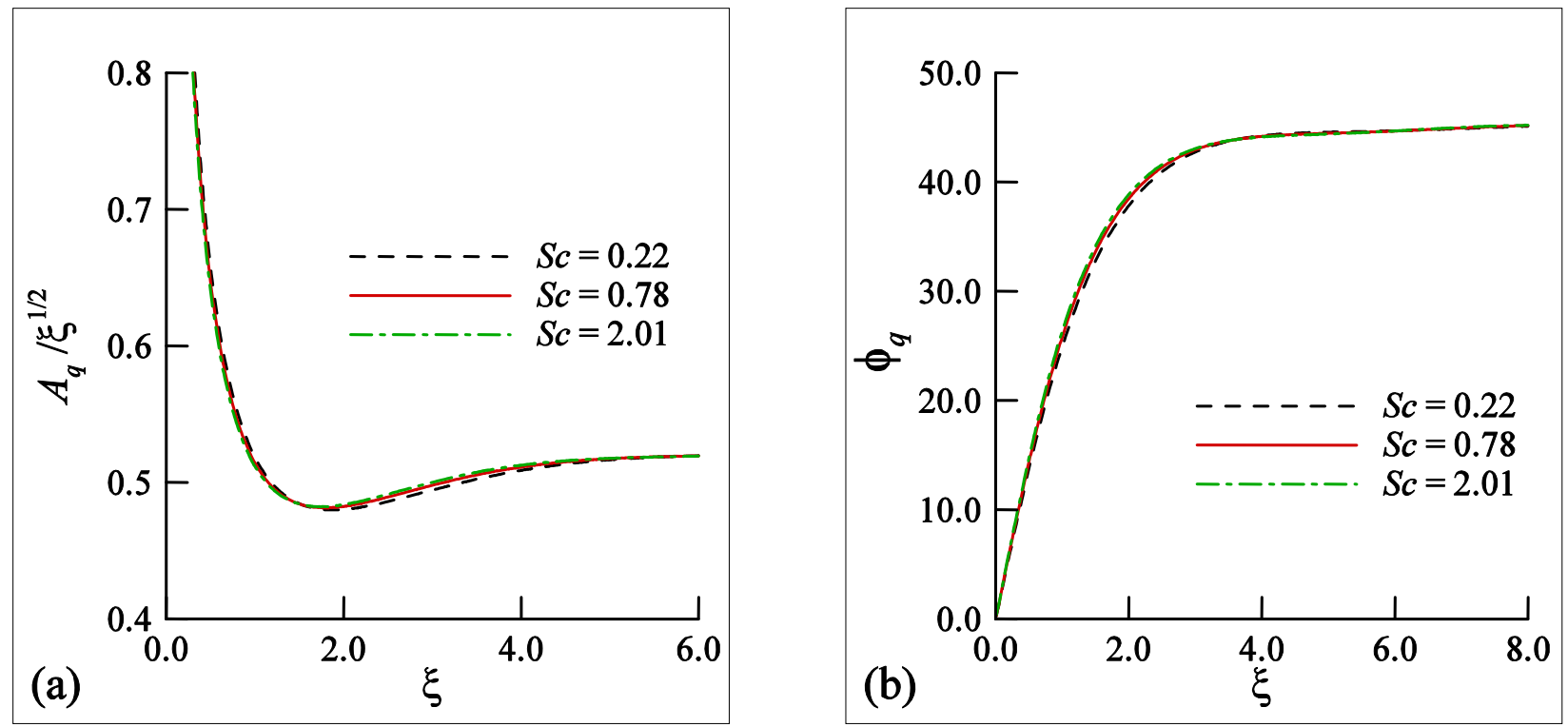

Fig.8. (a) Amplitude and (b) phase angle of heat transfer for different values of $\mathrm{Sc}$ while $\mathrm{Pr}=0.72, \mathrm{Ri}=$ $2.0, w=0.5, R_{d}=0.5, \Delta=0.5$. 

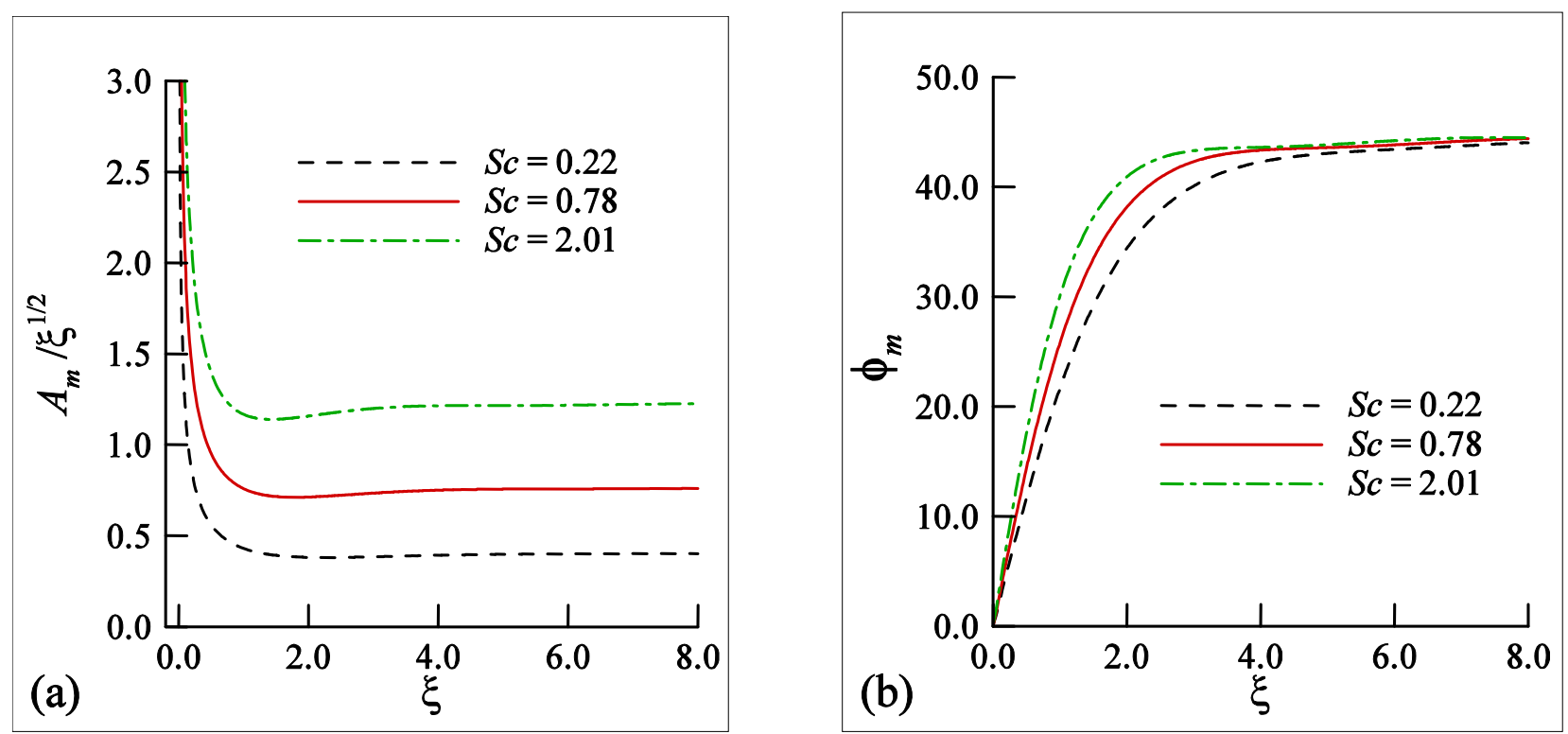

Fig.9. (a) Amplitude and (b) phase angle of mass transfer for different values of $S c$ while $\operatorname{Pr}=0.72, \mathrm{Ri}=$ $2.0, w=0.5, R_{d}=0.5, \Delta=0.5$.

The Schmidt number effects on the amplitudes and phase angles of skin friction, heat transfer and species mass transfer are presented in Figs 7-9. These figures show that the amplitudes of skin friction increase but the phase angles decrease with small values of Sc. This is due to the fact that the Schmidt number becomes high owing to either an increase of the kinematic viscosity or a decrease of the mass diffusivity of the fluid, and the increase of the skin friction is the result of this change in the fluid property. The changing of Sc has no strong effect on the amplitudes and phase angles of heat transfer although it has a strong effect on the amplitudes and phase angles of species mass transfer. As the value of Sc increases, the amplitudes and phase angles of mass transfer increase. This is because the Schmidt number, Sc, indicates the physical properties of the diffusing species.
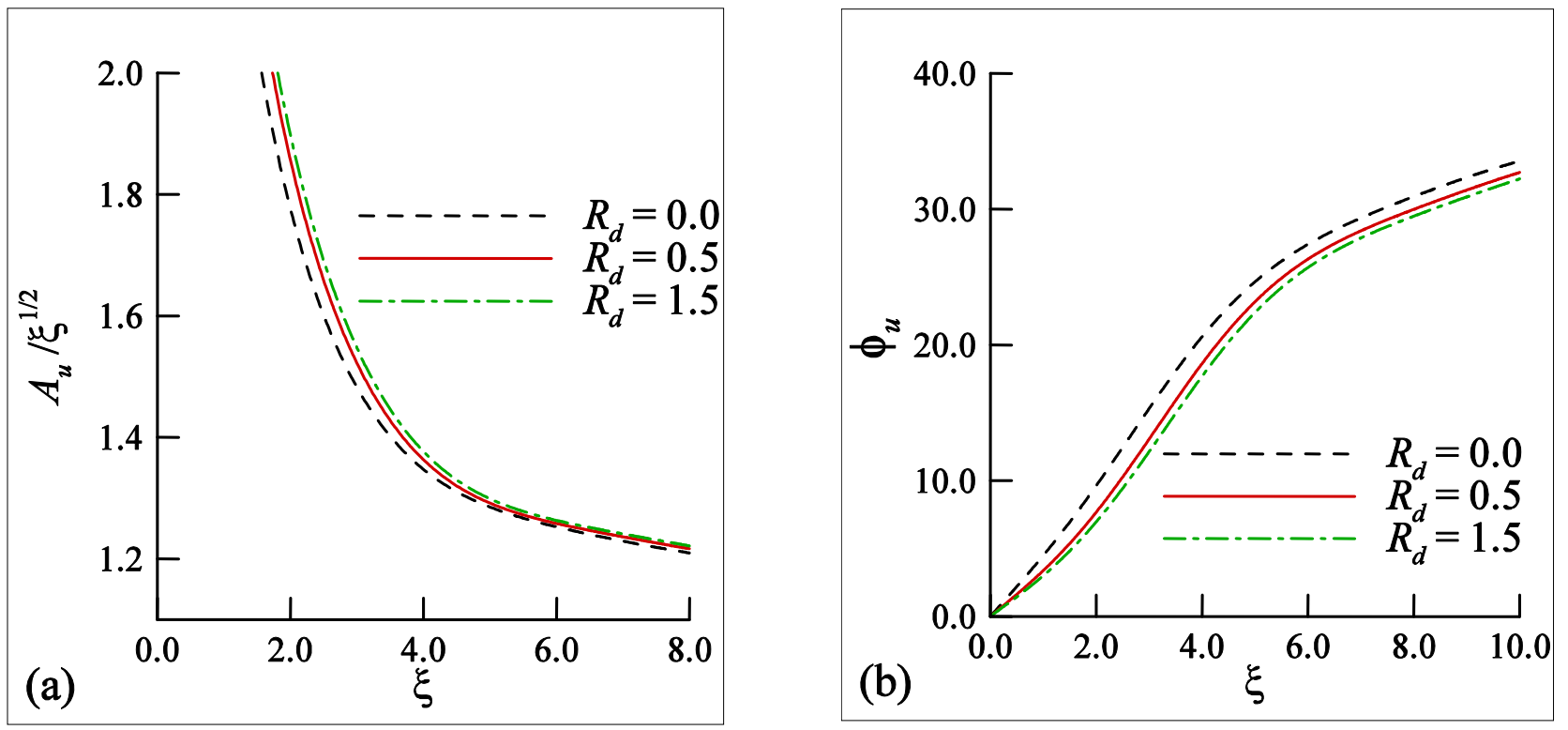

Fig10. (a) Amplitude and (b) phase angle of skin friction for different values of $R_{d}$ while $\operatorname{Pr}=0.72, \mathrm{Ri}=2.0$, $w=0.5, \mathrm{Sc}=0.22, \Delta=0.5$. 

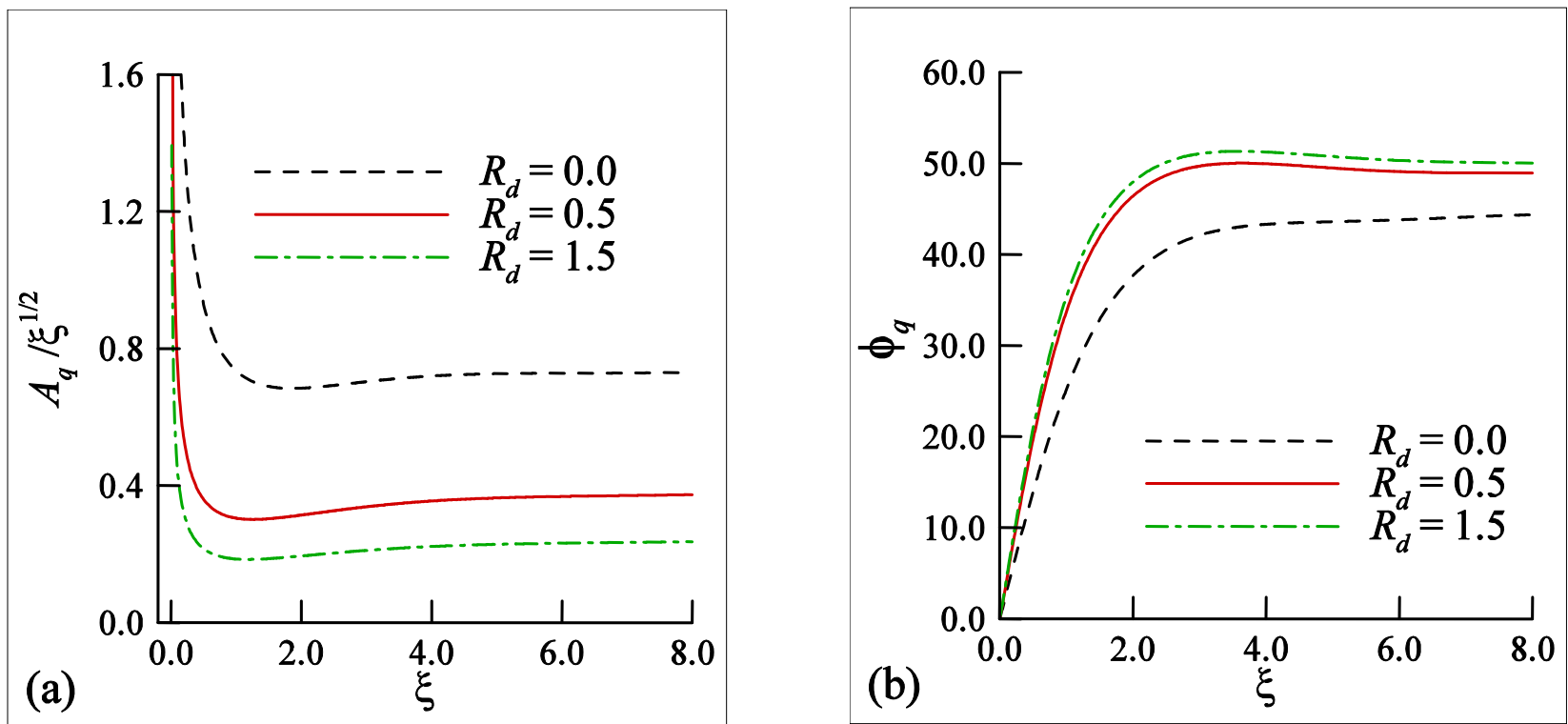

Fig.11. (a) Amplitude and (b) phase angle of heat transfer for different values of $R_{d}$ while $\operatorname{Pr}=0.72, \mathrm{Ri}=$ $2.0, w=0.5, \mathrm{Sc}=0.22, \Delta=0.5$.

The effects of varying the conduction-radiation parameter $R_{d}$ on the amplitudes and phase angles of skin friction, heat transfer and mass transfer are shown in Figs 10-12. While $R_{d}$ increases, the amplitudes of skin friction increase, but the phase angles decrease. It should be noted that the change of the amplitudes and phase angles is more profound when radiation dominates heat conduction (i.e., $R_{d}$ is large). It is obvious that an increase in the radiation parameter $R_{d}$ results in decreasing amplitudes but in increasing phase angles of heat transfer within the boundary layer. This is because the small $R_{d}$ values correspond to an increased dominance of conduction over radiation. Moreover the changing of $R_{d}$ has no strong effect on the amplitudes and phase angles of species mass transfer although it has a strong effect on the amplitudes and phase angles of heat transfer. As the value of $R_{d}$ increases, the amplitudes of mass transfer increase but the phase angles decrease.
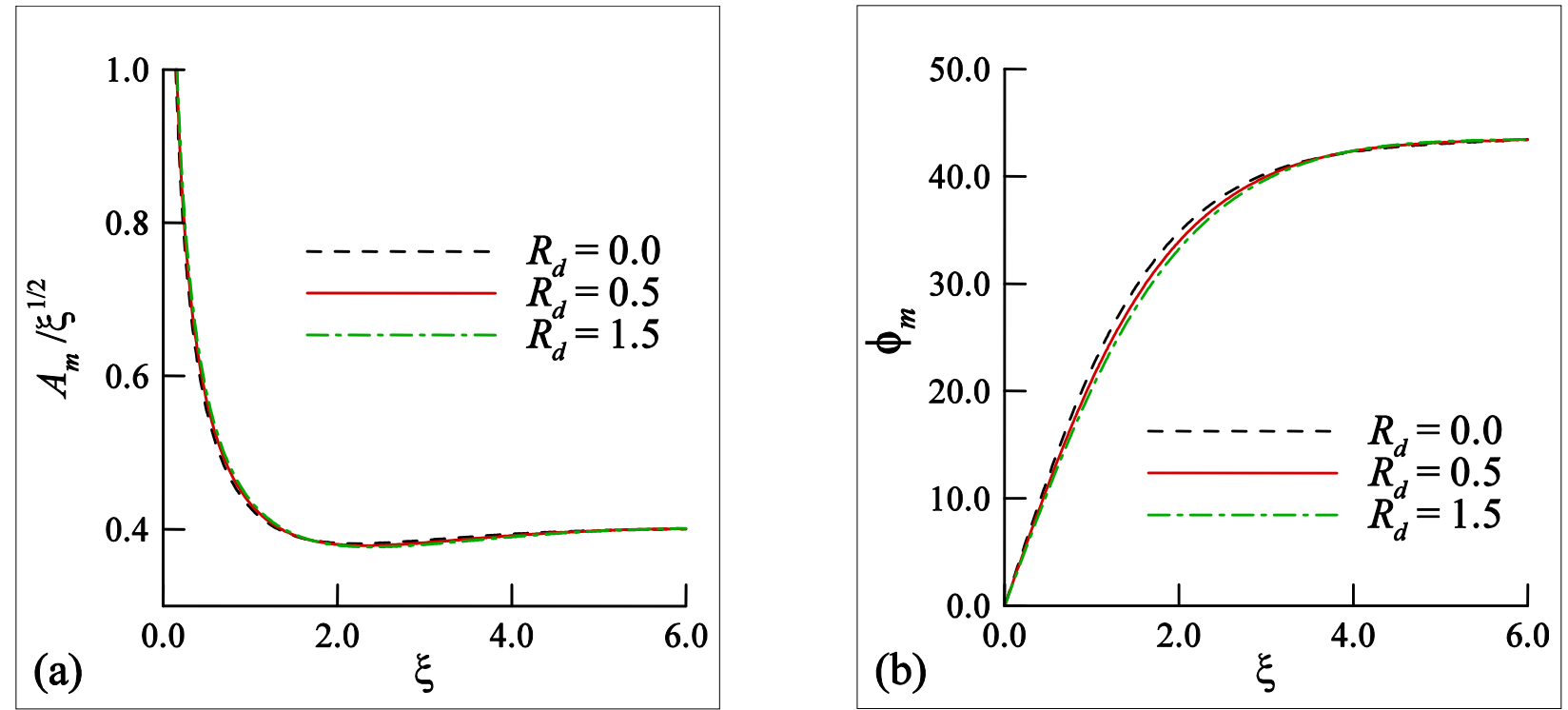

Fig.12. (a) Amplitude and (b) phase angle of mass transfer for different values of $R_{d}$ while $\operatorname{Pr}=0.72$, $\mathrm{Ri}=2.0, w=0.5, \mathrm{Sc}=0.22, \Delta=0.5$. 

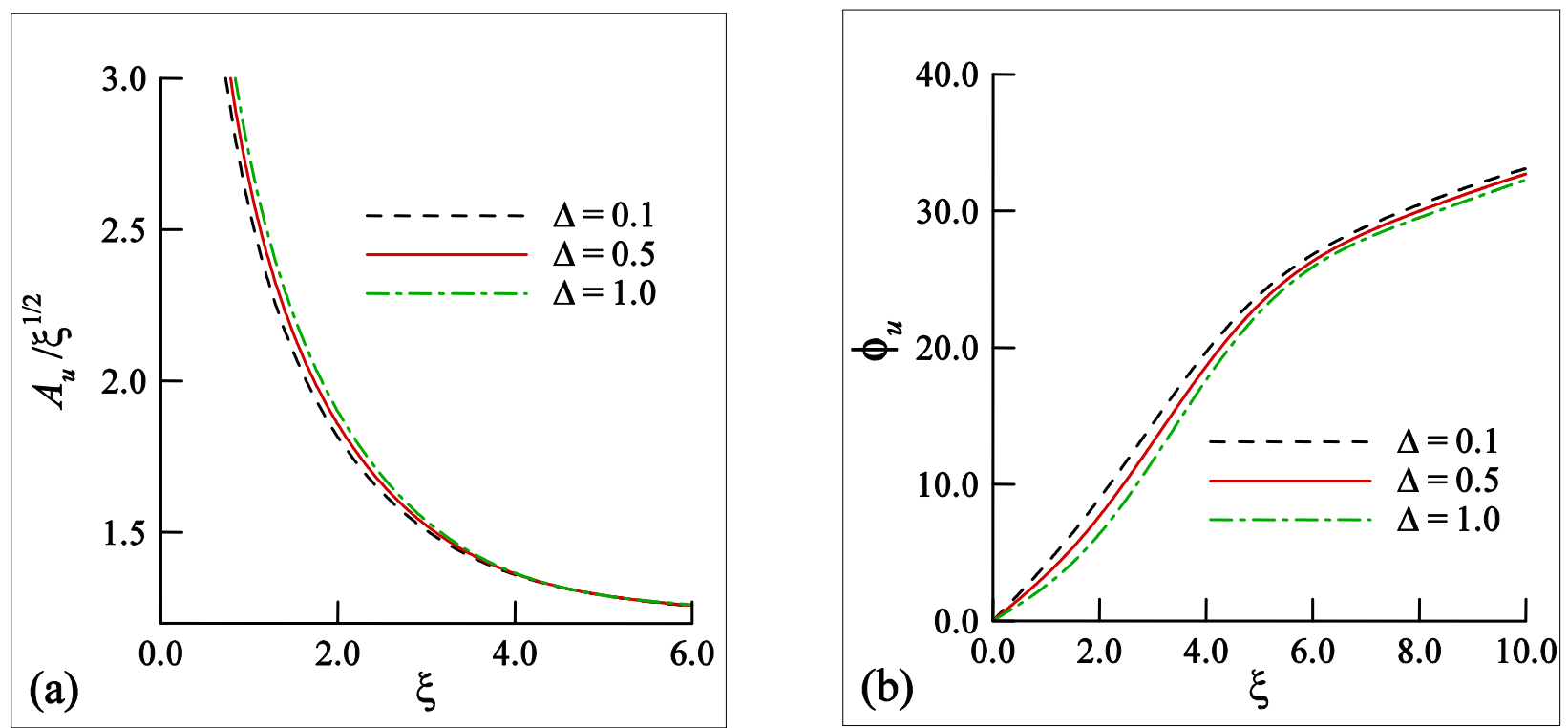

Fig.13. (a) Amplitude and (b) phase angle of skin friction for different values of $\Delta$ while $\operatorname{Pr}=0.72$, $\mathrm{Ri}=2.0, w=0.5, \mathrm{Sc}=0.22, R_{d}=0.5$.
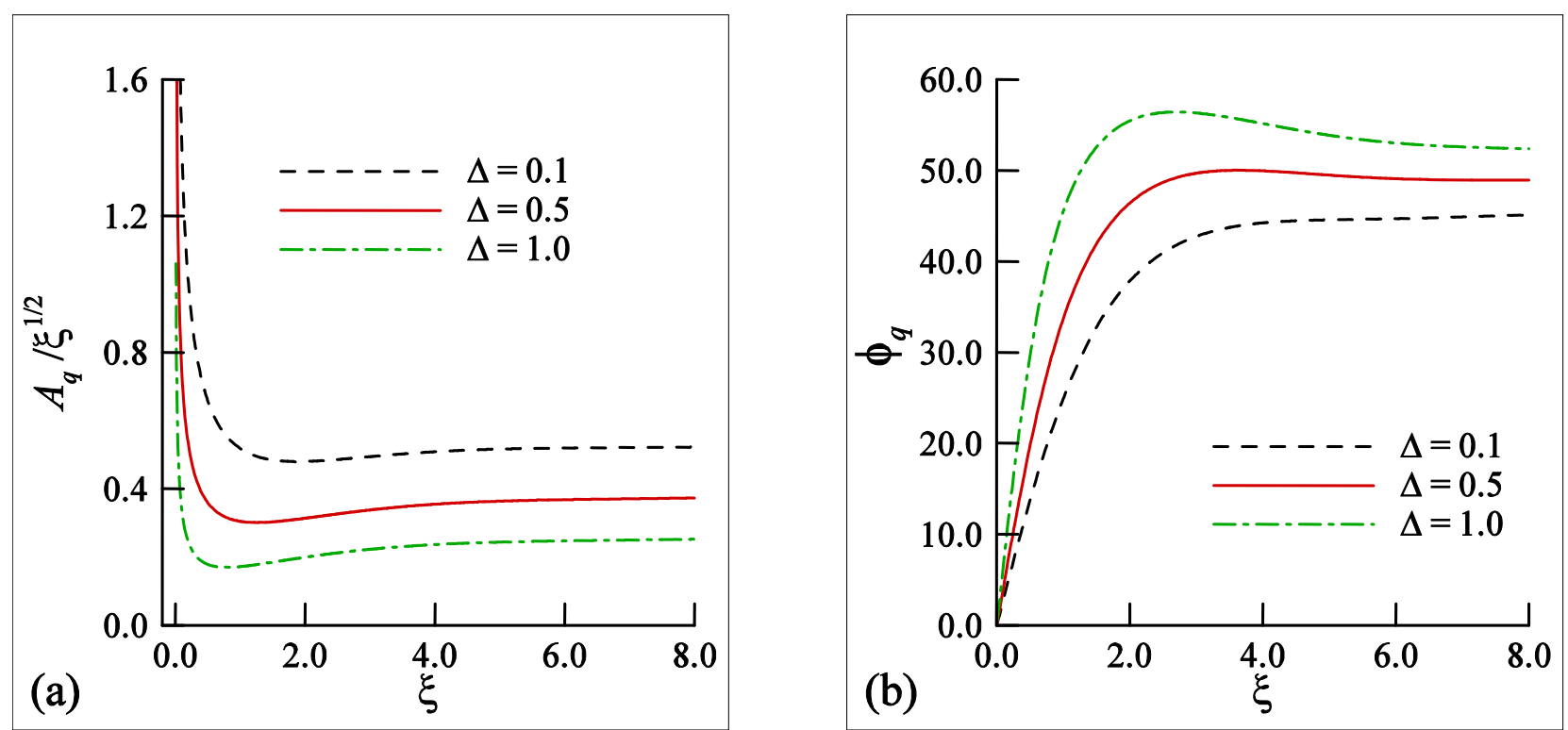

Fig.14. (a) Amplitude and (b) phase angle of heat transfer for different values of $\Delta$ while $\operatorname{Pr}=0.72$, $\mathrm{Ri}=2.0, w=0.5, \mathrm{Sc}=0.22, R_{d}=0.5$. 

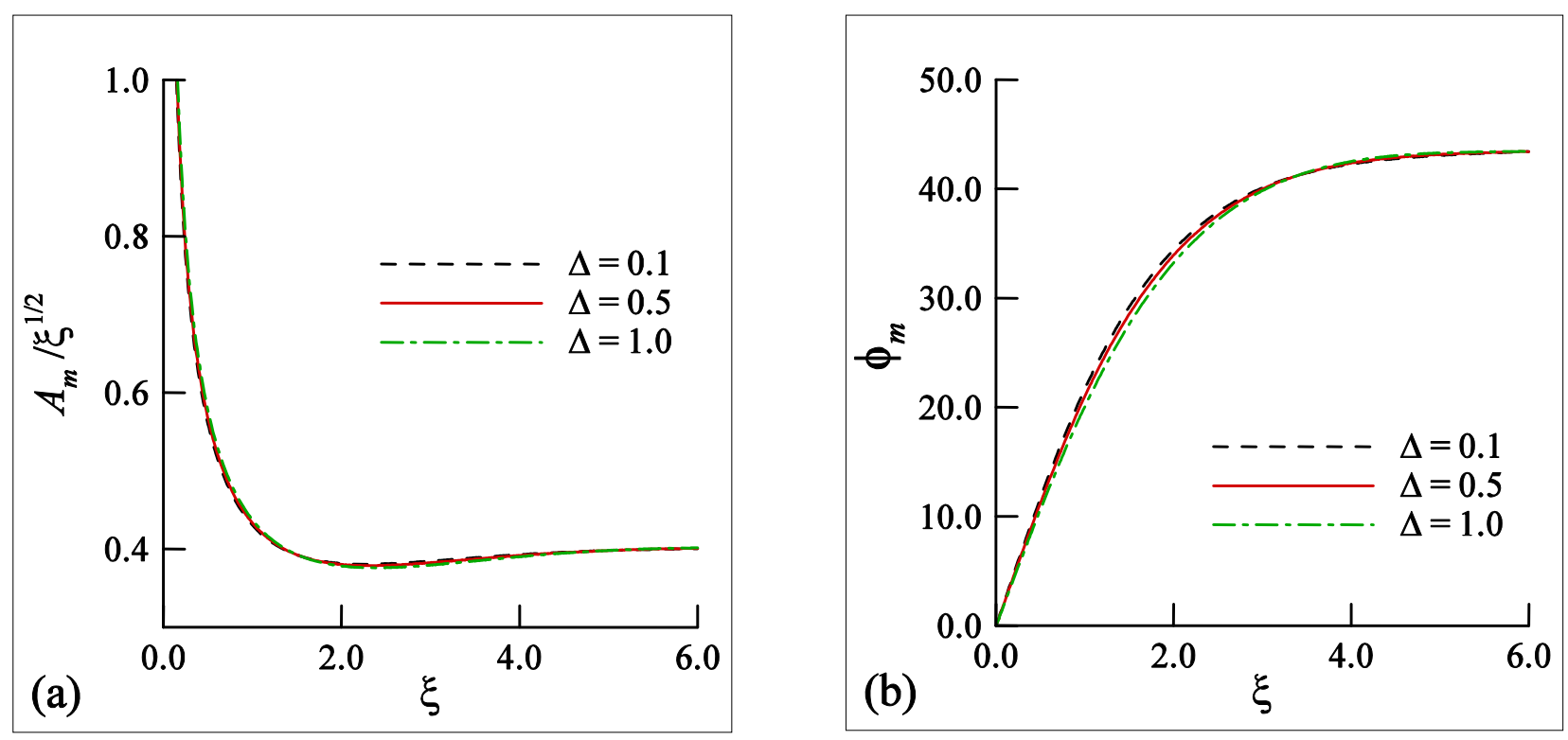

Fig.15. (a) Amplitude and (b) phase angle of mass transfer for different values of $\Delta$ while $\operatorname{Pr}=0.72$, $\mathrm{Ri}=2.0, w=0.5, \mathrm{Sc}=0.22, R_{d}=0.5$.

Figures 13-15 depict the influences of changing the surface temperature parameter $\Delta$ on the amplitudes and phase angles of skin friction, heat transfer and mass transfer. The figures indicate that the amplitudes of skin friction and mass transfer increase but the phase angles decrease with increasing values of the surface temperature parameter $\Delta$. However it is seen from Fig. 15 that the amplitudes of the rate of heat transfer decrease but the phase angles increase when the surface temperature parameter increases.

\subsection{Effects of different physical parameters on transient skin friction, heat transfer and mass transfer}

In this section, the effects of the physical parameter are discussed in terms of the transient skin friction, heat transfer and mass transfer which are determined using the following relations

$$
\begin{aligned}
& \tau=\tau_{s}+\varepsilon A_{u} \cos \left(\omega t+\phi_{u}\right), \\
& q=q_{s}+\varepsilon A_{q} \cos \left(\omega t+\phi_{q}\right), \\
& m=m_{s}+\varepsilon A_{m} \cos \left(\omega t+\phi_{m}\right)
\end{aligned}
$$

where $\tau_{s}=f^{\prime \prime}(0), q_{s}=-\theta^{\prime}(0)$ and $m_{s}=-\varphi^{\prime}(0)$ are, respectively, the steady-state skin friction, heat transfer and mass transfer. 

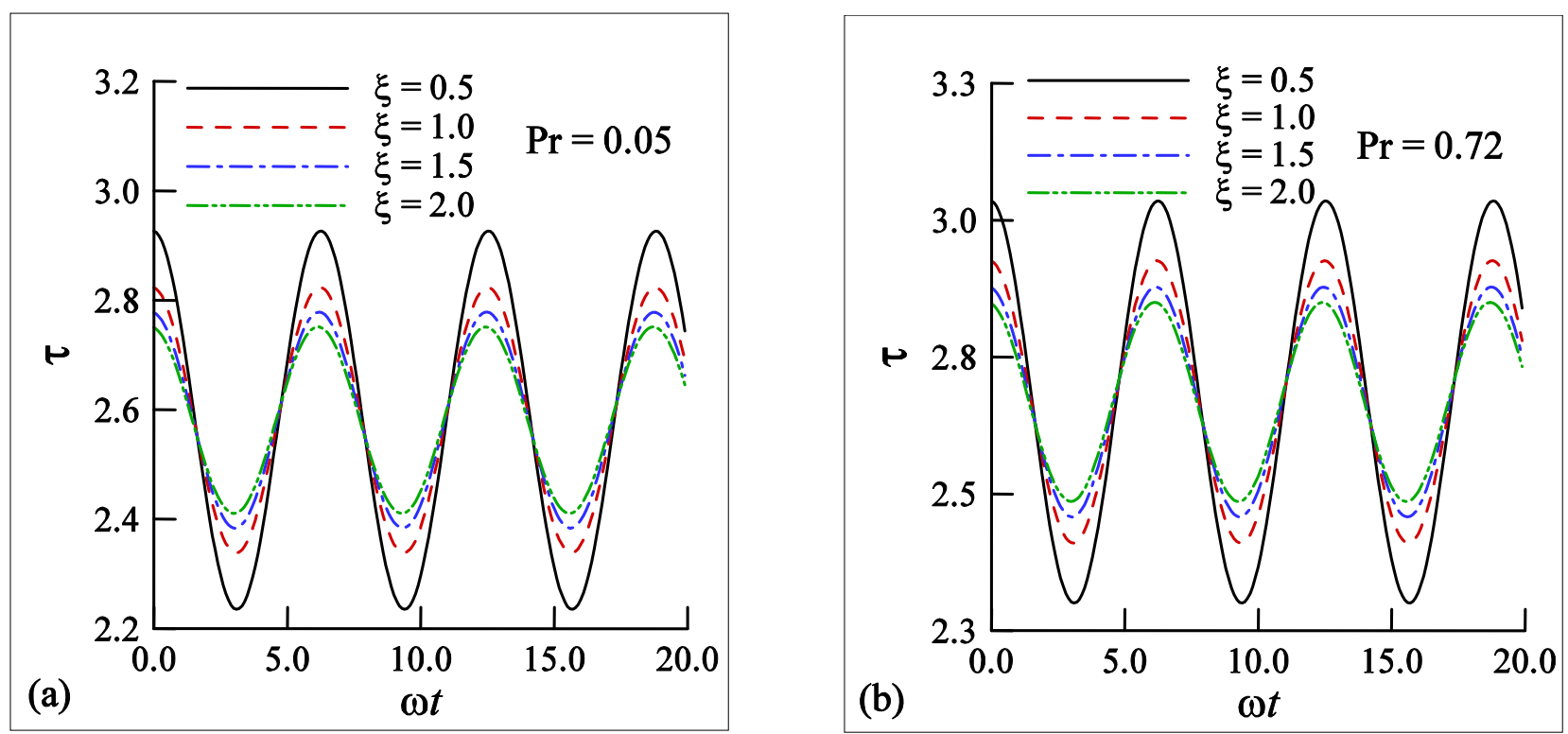

Fig.16. Numerical values of transient skin friction for different values of $\xi$ against $\omega t$ while $\mathrm{Ri}=2.0, w=0.5$, $R_{d}=0.5, \Delta=0.5, \mathrm{Sc}=0.22, \operatorname{Pr}=0.05$ and $\operatorname{Pr}=0.72$.
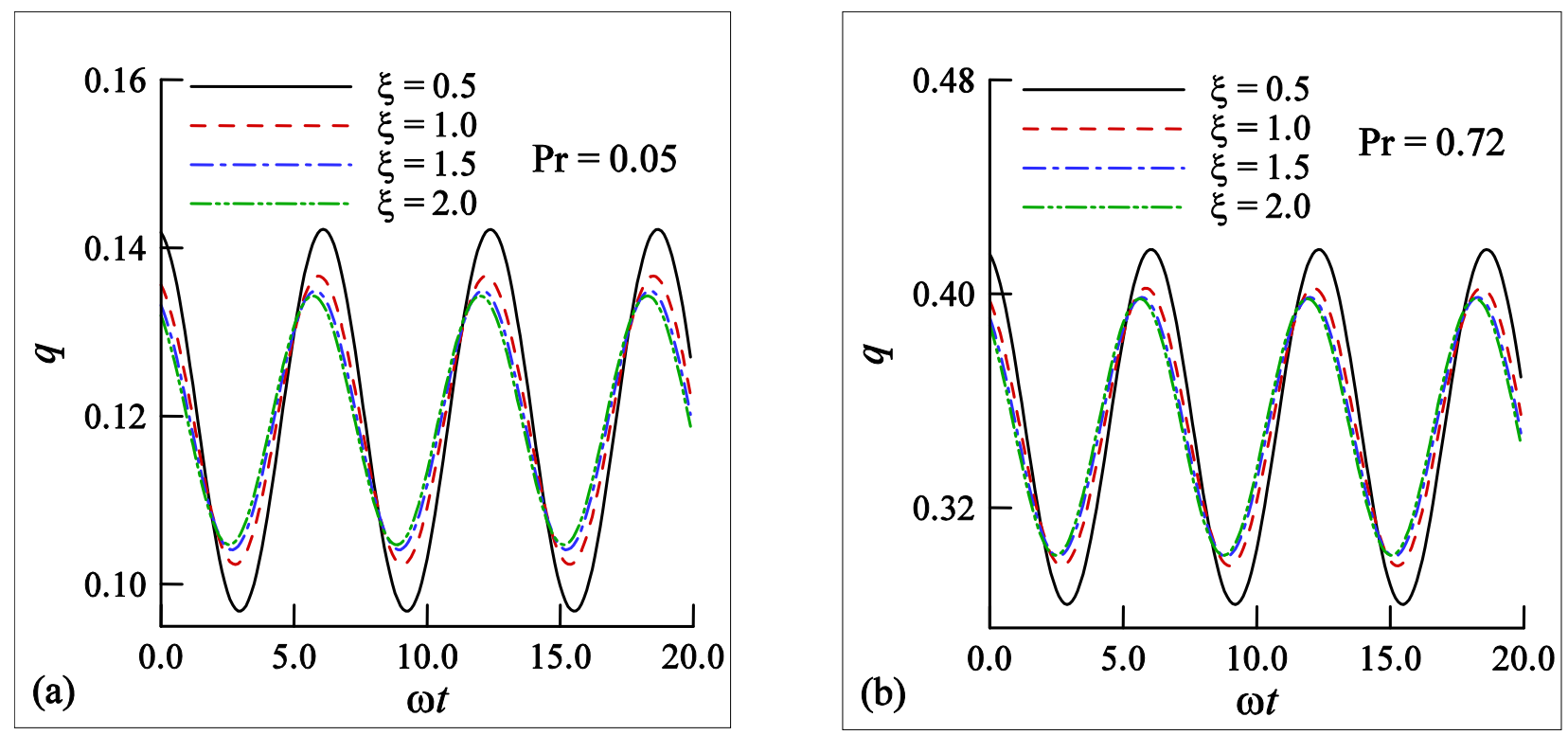

Fig.17. Numerical values of transient heat transfer for different values of $\xi$ against $\omega t$ while $\mathrm{Ri}=2.0, w=0.5$, $R_{d}=0.5, \Delta=0.5, \mathrm{Sc}=0.22, \operatorname{Pr}=0.05$ and $\operatorname{Pr}=0.72$. 

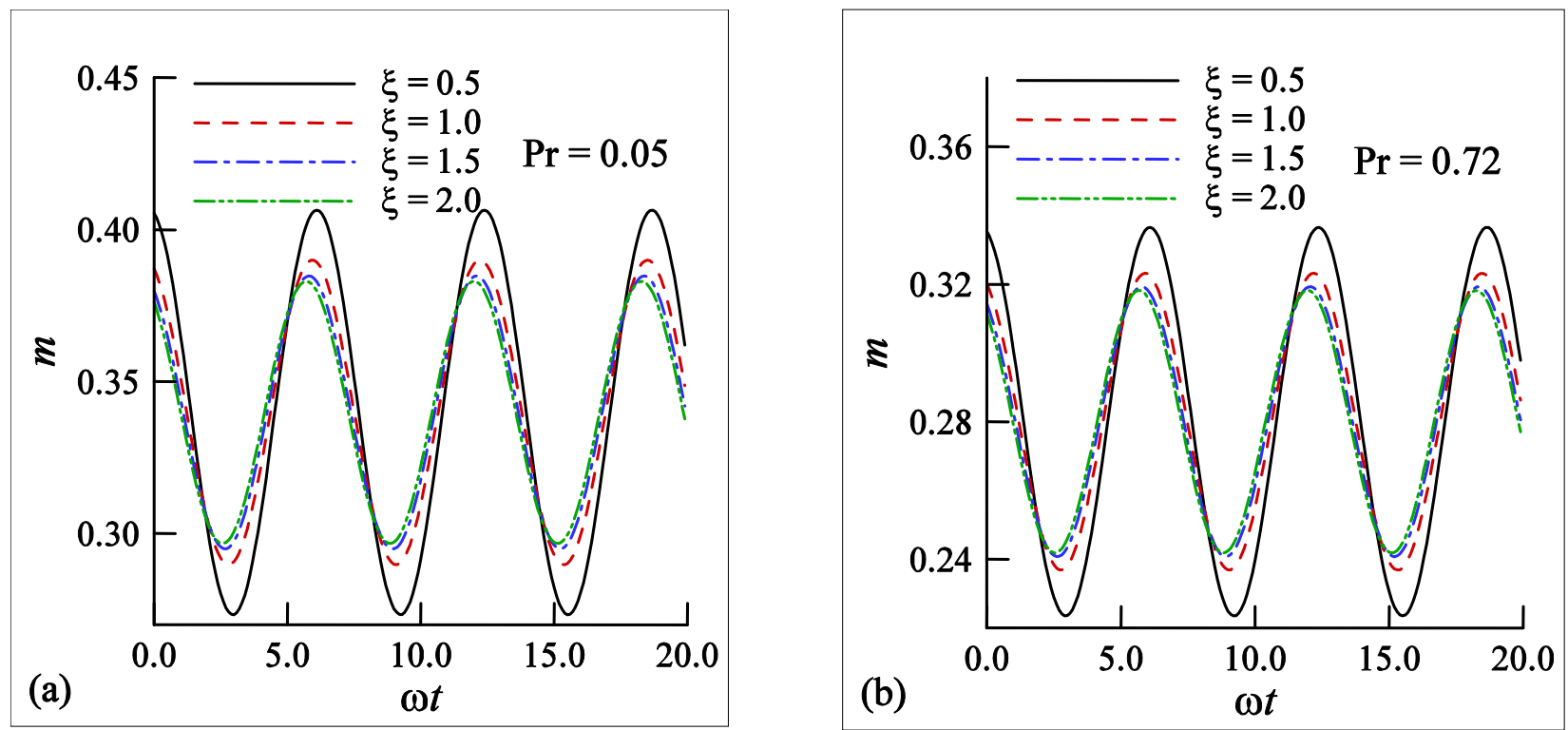

Fig.18. Numerical values of transient mass transfer for different values of $\xi$ against $\omega t$ while $\mathrm{Ri}=2.0, w=$ $0.5, R_{d}=0.5, \Delta=0.5, \mathrm{Sc}=0.22, \operatorname{Pr}=0.05$ and $\operatorname{Pr}=0.72$.

The effects of varying the Prandtl number, Pr, on the transient skin friction, heat transfer and mass transfer are shown in Figs 16, 17 and 18, respectively, for $\operatorname{Pr}=0.05$ and $\operatorname{Pr}=0.72$. Results suggest that the amplitudes of oscillation of the transient skin friction, $\tau$, and heat transfer, $q$, increase but that of mass transfer, $m$, decrease with an increase of the Prandtl number, Pr. The amplitudes of oscillation die down slowly from the leading edge to the downstream region, that is, with increasing $\xi$. However, this change in amplitudes oscillation is accelerated for higher values of the Prandtl number.
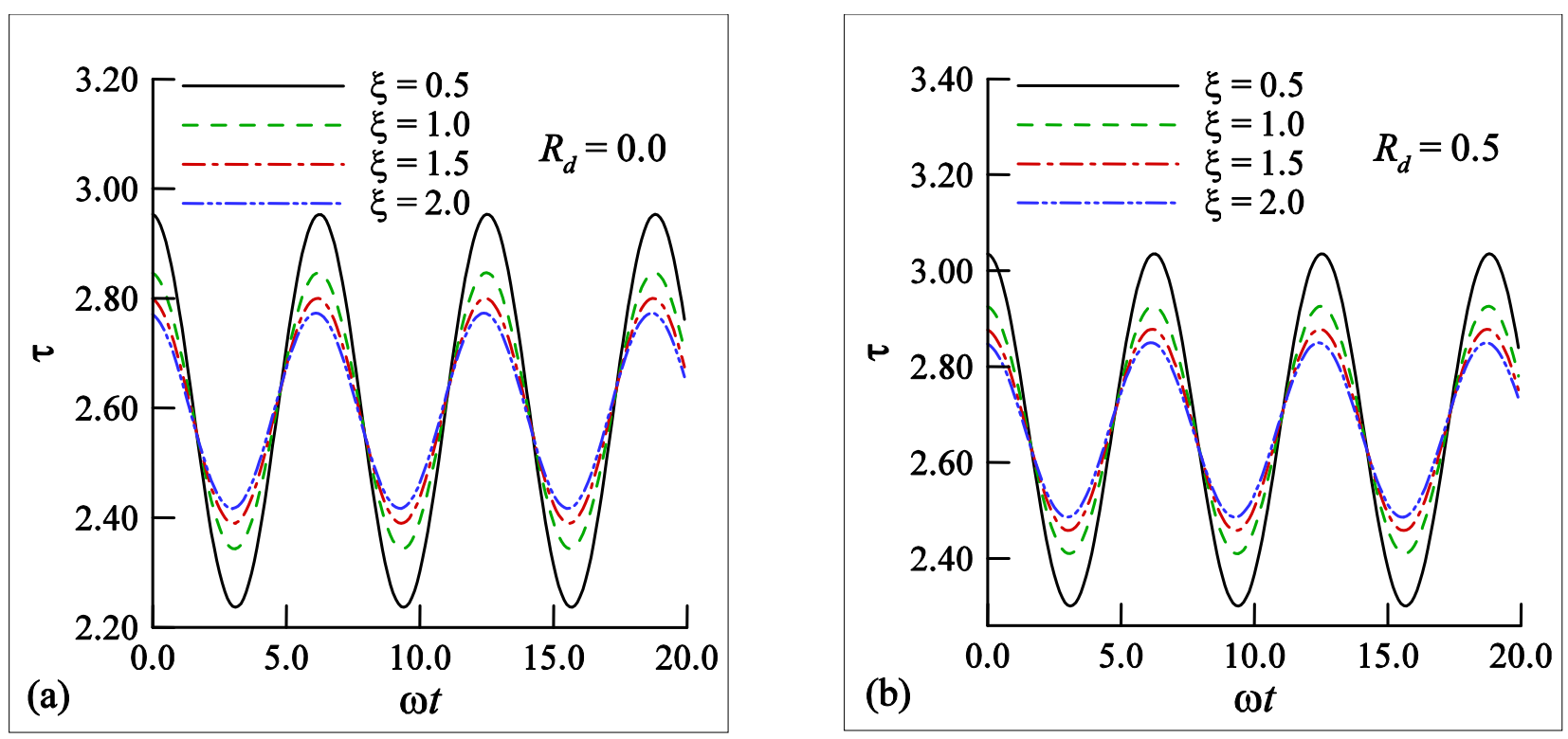

Fig.19. Numerical values of transient skin friction for different values of $\xi$ against $\omega t$ while $\operatorname{Pr}=0.72, \operatorname{Ri}=$ $2.0, w=0.5, \Delta=0.5, \mathrm{Sc}=0.22, R_{d}=0.0$ and $R_{d}=0.5$. 

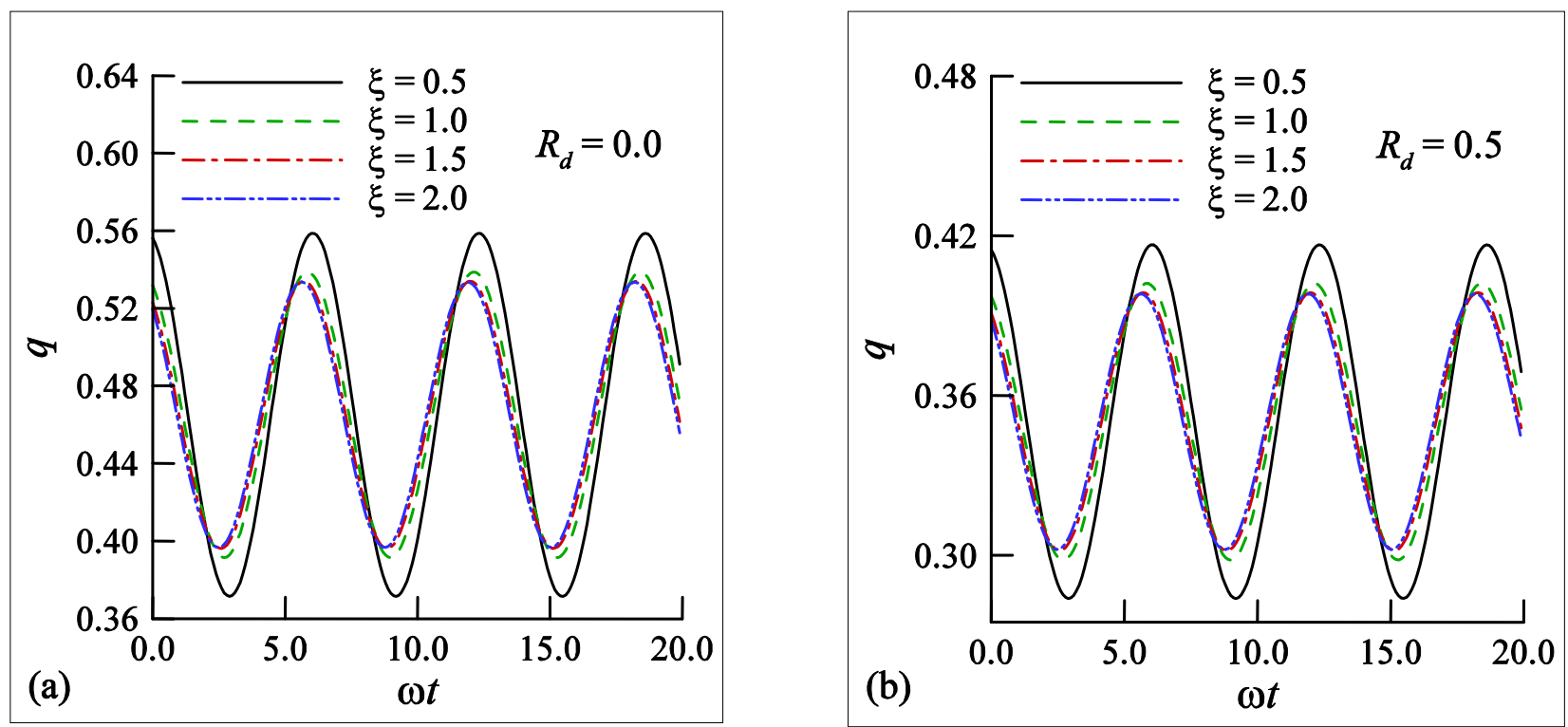

Fig.20. Numerical values of transient heat transfer for different values of $\xi$ against $\omega t$ while $\operatorname{Pr}=0.72, \mathrm{Ri}=$ $2.0, w=0.5, \Delta=0.5, \mathrm{Sc}=0.22, R_{d}=0.0$ and $R_{d}=0.5$.
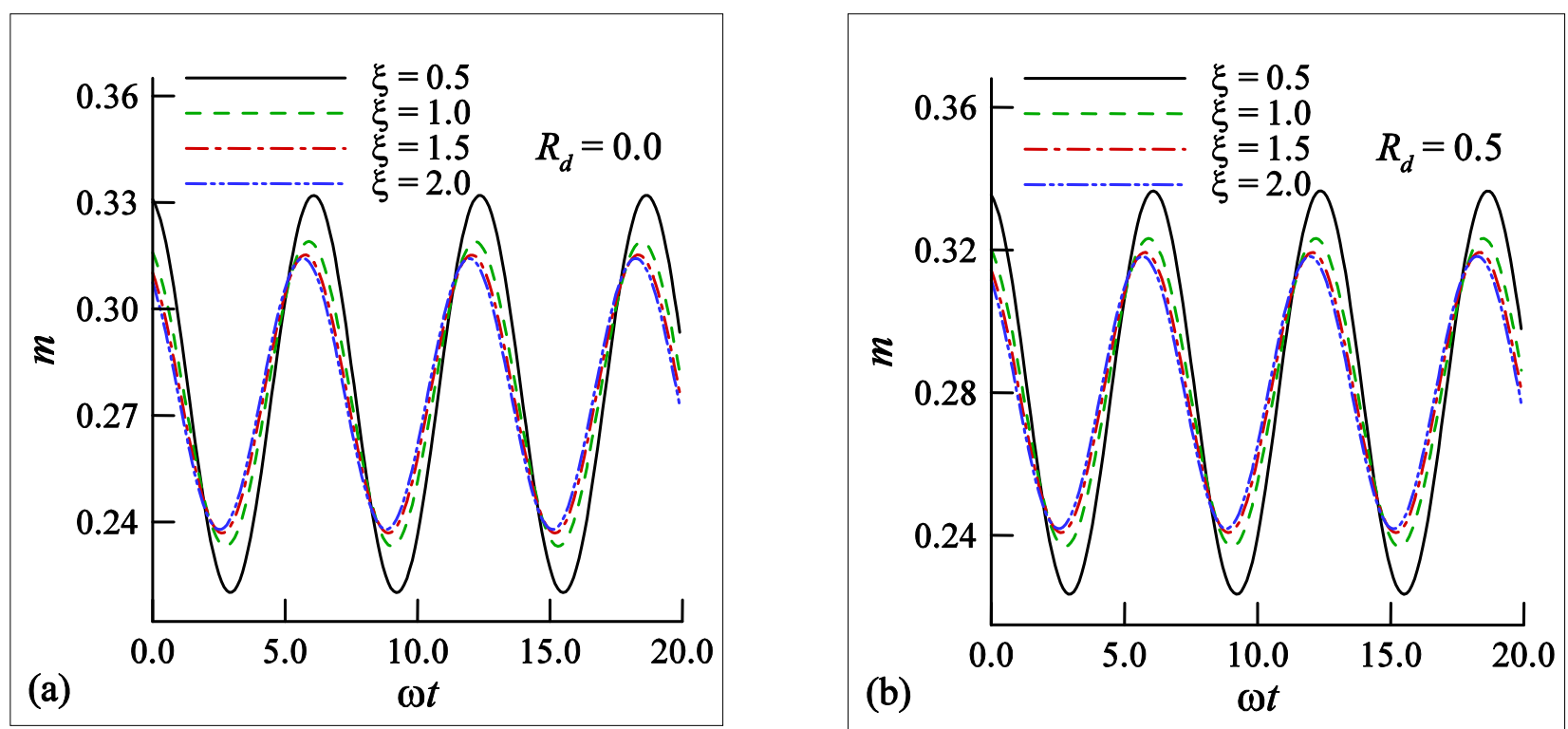

Fig.21. Numerical values of transient mass transfer for different values of $\xi$ against $\omega t$ while $\operatorname{Pr}=0.72, \mathrm{Ri}=$ $2.0, w=0.5, \Delta=0.5, S c=0.22, R_{d}=0.0$ and $R_{d}=0.5$.

In Figs 19-21, numerical values of the transient skin-friction coefficient, heat transfer coefficient and transient mass transfer coefficient have been presented, respectively, against $\omega t$, taking $\operatorname{Pr}=0.72, \mathrm{Ri}=2.0, w$ $=0.5, \Delta=0.5, \mathrm{Sc}=0.22, R_{d}=0.0$ and $R_{d}=0.5$. From these figures it is seen that the amplitudes of oscillation of the transient skin-friction increase but these of transient heat transfer decrease when $R_{d}$ increases. On the other hand, the amplitudes of oscillation of the transient mass transfer display a small increment for increasing the values of $R_{d}$. This may be explained by noting that an increase in $R_{d}$ means an increase in internal heat due to radiation in the boundary layer, which leads to an increase in the momentum boundary layer thickness while the thermal boundary layer thickness is reduced. This consequently causes an 
increase in the transient skin-friction $\tau$, the transient mass transfer $m$ and a decrease in the transient heat transfer $q$.

\section{Conclusions}

The mixed convection boundary layer flow of a viscous incompressible fluid past a vertical wedge has been investigated taking into account the effect of thermal radiation. The skin friction, heat transfer and mass transfer are found to increase with an increase of the Richardson number. The higher Prandtl number enhances the skin friction and the heat transfer but reduces the mass transfer. Also, the conduction-radiation parameter and the surface temperature parameter significantly diminish the rate of heat transfer while their effects on the skin friction and mass transfer are rather weak. However, mass transfer is strongly dependent on the Schmidt number. For higher Prandtl number the amplitudes of oscillation of transient skin friction and heat transfer increase but these of mass transfer decrease. In addition, an increase in the conduction-radiation parameter causes an increase in the transient skin-friction and mass transfer but a decrease in the transient heat transfer.

\section{Nomenclature}

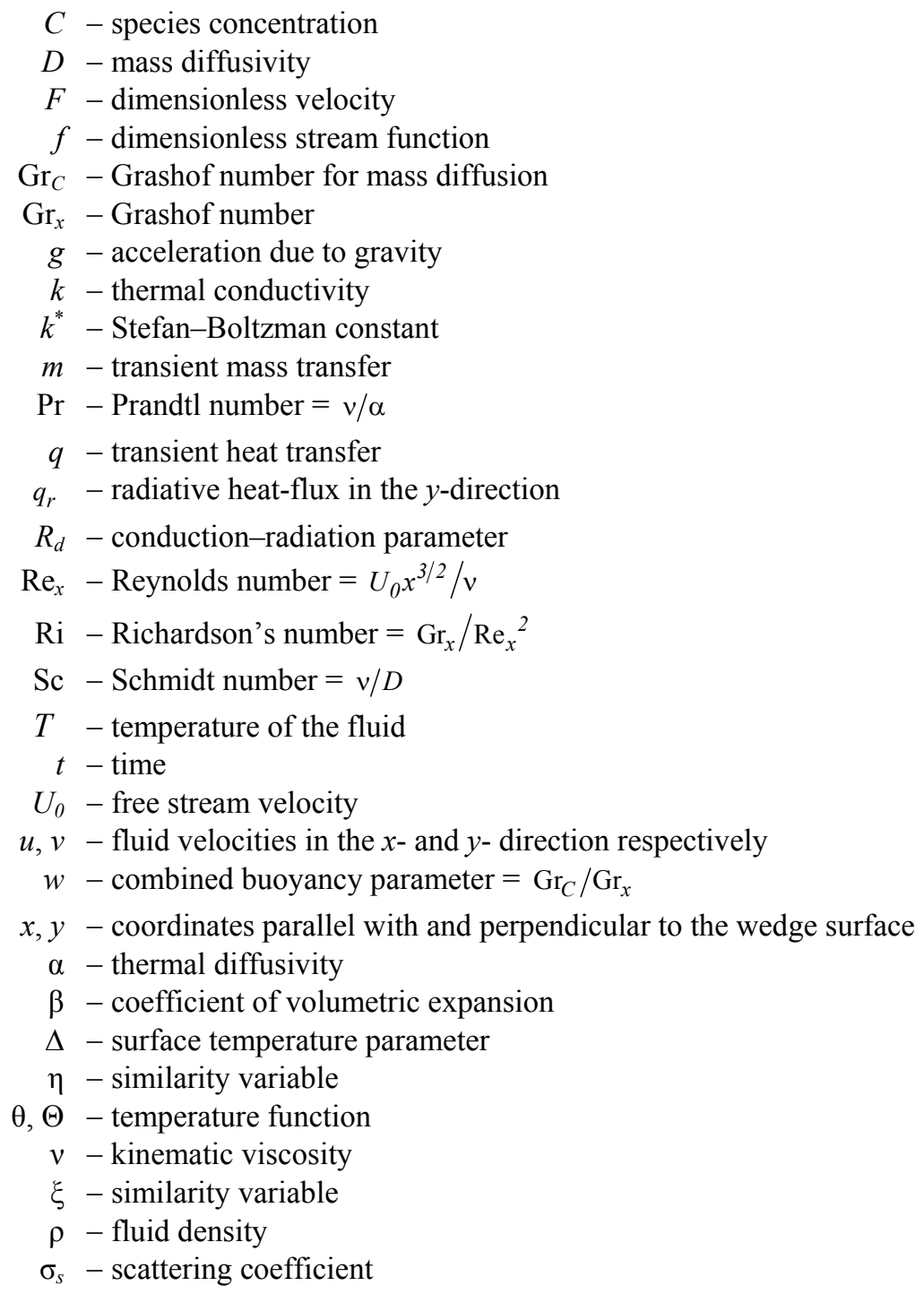


$\tau-$ transient skin friction

$\psi-$ stream function

$\varphi, \Phi-$ species concentration function

$\omega$ - amplitude of oscillation

\section{Subscripts}

$C$ - based on species concentration level

$s$ - refers to the steady state

$T$ - based on temperature level

$w$ - refers to the wall

$\infty$ - ambient condition

\section{Superscript}

' - differentiation with respect to $Y$ or $\eta$

\section{References}

[1] Lighthill M.J. (1954): The response of laminar skin friction and heat transfer to fluctuations in the stream velocity. - Proc. R. Soc., Lond. A., vol.224, pp.1-23.

[2] Sing P.J., Roy S. and Ravindran R. (2009): Unsteady mixed convection flow over a vertical wedge. - Int. J. Heat Mass Transf., vol.52, pp.415-421.

[3] Yih K.A. (1998): Uniform suction/blowing effects on forced convection about a wedge. - Acta Mech., vol.128, pp.173-181.

[4] Watanabe T. (1990): Thermal boundary-layer over a wedge with uniform suction or injection in forced flow. Acta Mech., vol.83, pp.119-126.

[5] Kafoussias N.G. and Nanousis N.D. (1997): Magnetohydrodynamic laminar boundary layer flow over a wedge with suction or injection. - Can. J. Phys., vol.75, pp.733-745.

[6] Anjali Devi S.P. and Kandasamy R. (2001): Effects of thermal stratification on laminar boundary layer flow over a wedge with suction or injection. - Mech. Res. Commun., vol.28, pp.349-354.

[7] Gersten K. (1965): Heat transfer in laminar boundary layers with oscillating outer flow. - AGARBograph, vol.97, pp.423-475.

[8] Kumari M. and Gorla R.S.R. (1997): Combined convection along a non-isothermal wedge in a porous medium. Heat Mass Transf., vol.32, pp.393-398.

[9] Hossain M.A., Munir M.S., Hafiz M.Z. and Takhar H.S. (2000): Flow of a viscous incompressible fluid of temperature dependent viscosity past a permeable wedge with uniform surface heat flux. - Heat Mass Transf., vol.36, pp.333-341.

[10] Kumari M., Takhar H.S. and Nath G. (2001): Mixed convection flow over a vertical wedge embedded in a highly porous medium. - Heat Mass Transf., vol.37, pp.139-146.

[11] Kandasamy R., Muhaimin I. and Khamis A.B. (2009): Thermophoresis and variable viscosity effects on MHD mixed convective heat and mass transfer past a porous wedge in the presence of chemical reaction. - Heat Mass Transf., vol.45, pp.703-712.

[12] Uddin Z., Kumar M. and Harmand S. (2014): Influence of thermal radiation and heat generation/absorption on MHD heat transfer flow of a micropolar fluid past a wedge considering hall and ion slip currents. - Thermal Sci., vol.18, No.2, pp.489-502.

[13] Yih K.A. (2001): Radiation effect on mixed convection over an isothermal wedge in porous media, the entire regime. - Heat Transf. Eng., vol.22, pp.26-32. 
[14] Al-Odat M.Q., Al-Hussien F.M.S. and Damseh R.A. (2005): Influence of radiation on mixed convection over a wedge in non-Darcy porous medium. - Forsch Ing., vol.69, pp.209-215.

[15] Chamkha A.J., Mujtaba M., Quadri A. and Issa C. (2003): Thermal radiation effects on MHD forced convection flow adjacent to a non-isothermal wedge in the presence of heat source or sink. - Heat Mass Transf., vol.39, pp.305-312.

[16] Elbashbehy E.M.A. and Dimian M.F. (2001): Effect of radiation on the flow and heat transfer over a wedge with variable viscosity. - Applied Math. Comp., vol.132, pp.445-454.

[17] Butcher J.C. (1964): Implicit Runge-Kutta method. - Math. Com., vol.18, pp.50-55.

[18] Naschtsheim P.R. and Sweigert P. (1965): Satisfaction of asymptotic boundary conditions in numerical solution of systems of non-linear equation of boundary layer type. - NASA TN D-3004.

Received: April 10, 2016

Revised: March 13, 2017 